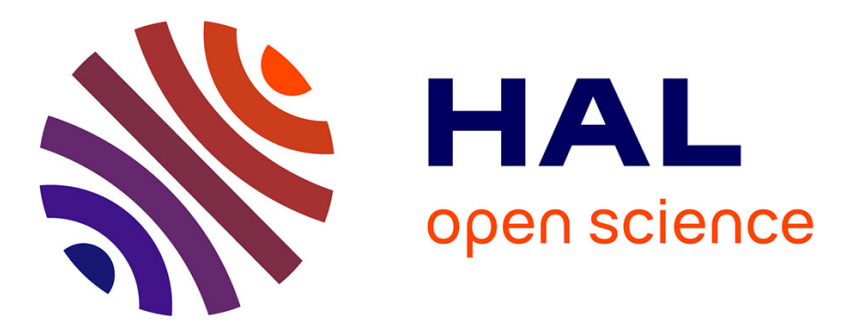

\title{
UV Excitations of Halons UV Excitations of Halons
}

Ljiljana Stojanović, Abdulrahman O Alyoubi, Saadullah G Aziz, Rifaat H

Hilal, Mario Barbatti

\section{To cite this version:}

Ljiljana Stojanović, Abdulrahman O Alyoubi, Saadullah G Aziz, Rifaat H Hilal, Mario Barbatti. UV Excitations of Halons UV Excitations of Halons. Journal of Chemical Physics, 2016, 145 (18), pp.184306. 10.1063/1.4967170 . hal-02288821

\section{HAL Id: hal-02288821 \\ https://hal-amu.archives-ouvertes.fr/hal-02288821}

Submitted on 16 Sep 2019

HAL is a multi-disciplinary open access archive for the deposit and dissemination of scientific research documents, whether they are published or not. The documents may come from teaching and research institutions in France or abroad, or from public or private research centers.
L'archive ouverte pluridisciplinaire HAL, est destinée au dépôt et à la diffusion de documents scientifiques de niveau recherche, publiés ou non, émanant des établissements d'enseignement et de recherche français ou étrangers, des laboratoires publics ou privés. 


\section{UV Excitations of Halons}

Ljiljana Stojanović, ${ }^{1, a)}$ Abdulrahman O. Alyoubi, ${ }^{2}$ Saadullah G. Aziz, ${ }^{2}$ Rifaat H.Hilal, ${ }^{2,3}$ and Mario Barbatti, b)

1) Aix Marseille Univ, CNRS, ICR, Marseille, France

${ }^{2}$ Chemistry Department, Faculty of Science, King Abdulaziz University, Jeddah B.O. 208203, Saudi Arabia

3) Chemistry Department, Faculty of Science, Cairo University, Giza, Egypt

In the present study, we examined the UV excitations of a newly introduced molecular set, Halons-9, composed of nine gaseous halon molecules. The performance of the density functional-based multi-reference configuration interaction method (DFT/MRCI) and time-dependent density functional theory with CAM-B3LYP functional (TDCAM-B3LYP) in the computation of singlet and triplet excited states of this set was evaluated against coupled-cluster with singles and doubles (CCSD). Excited states up to the corresponding ionization limits, including both localized and delocalized excitations, have been benchmarked. TD-CAM-B3LYP significantly underestimates excitation energies of the higher mixed valence-Rydberg and Rydberg states, with computed mean absolute deviations from the EOM-CCSD results 1.06 and $0.76 \mathrm{eV}$, respectively. DFT/MRCI gives a significantly better description of higher excited states, albeit still poor, compared to the TD-CAM-B3LYP. The mean absolute deviations of mixed valence-Rydberg and Rydberg states from the reference EOM-CCSD values are 0.66 and $0.47 \mathrm{eV}$, respectively. The performance of DFT/MRCI for description of strongly correlated states with valence-Rydberg mixing is still not satisfactory enough. On the other hand, oscillator strengths of most of singlet states obtained with both methods are close to the EOM-CCSD values. The largest deviations, occurring in the case of several high-lying multiconfigurational states, are of an order of magnitude.

Keywords: halons, excited states, benchmarking, DFT/MRCI, TD-DFT

\footnotetext{
${ }^{a)}$ Electronic mail: stojanovicmljiljana@gmail.com
}

b) Electronic mail: mario.barbatti@univ-amu.fr 
UV Excitations of Halons

\section{INTRODUCTION}

One of the main challenges in quantum chemistry is the reliable prediction of excitedstate properties. Numerous theoretical methods for computation of excited states have been introduced and assessed, among them several popular single-reference methods (e.g. the linear-response time-dependent density functional theory (TD-DFT) ${ }^{1,2}$ and coupledcluster-based methods), as well as multi-reference methods (e.g. the complete active space second-order perturbation theory $(\mathrm{CASPT} 2)^{3}$ and the multi-reference configuration interaction (MRCI) ${ }^{4}$ method). However, selecting an appropriate electronic structure method for excited state computation in a particular case, in terms of accuracy and computational cost, is not a straightforward problem.

A number of computational studies have been devoted to performance evaluation of different quantum chemistry methods for prediction of various molecular properties on different molecular sets. ${ }^{5-11}$ Since the focus of this study is on excited states, we here mention few well-established molecular sets, which stand out among numerous sets used in methods assessments. The molecular set by Thiel and his co-workers ${ }^{5}$, an assembly of 28 small representative organic molecules, is one of the most widely used molecular sets. It has been employed for evaluation of excited states of several multi- and single-reference methods. ${ }^{5-8}$ Furthermore, Grimme and his co-workers introduced several molecular sets ${ }^{9-12}$. The first of them ${ }^{9}$ comprises 14 middle-sized to large molecules, including organic and inorganic molecules, where the studied excited states included the lowest-lying localized and Rydberg states. The molecular set composed of 14 small- to medium-sized molecules, introduced by Leang et al., was used for benchmarking of TD-DFT with various density functionals ${ }^{13}$. Winter at al. introduced a molecular set of 66 organic molecules, including porphine derivatives, polycyclic aromatic hydrocarbons, heterocyclic organic compounds and aromatic alcohols, and assessed several single-reference methods for the excited state computations ${ }^{14}$. By inspecting these and other prominent molecular sets, we noticed that a large field of halo-organic compounds remained not properly covered by any of them. This motivated us to introduce a new molecular set, Halons-9, composed of nine halon molecules: bromomethane $\left(\mathrm{CH}_{3} \mathrm{Br}\right)$, dichlorofluoromethane $\left(\mathrm{CHCl}_{2} \mathrm{~F}\right)$, bromodifluoromethane $\left(\mathrm{CHBrF}_{2}\right)$, bromochlorodifluoro-methane $\left(\mathrm{CBrClF}_{2}\right)$, dichlorodifluoromethane $\left(\mathrm{CCl}_{2} \mathrm{~F}_{2}\right)$, bromotrifluoromethane $\left(\mathrm{CBrF}_{3}\right)$, tetrafluoromethane $\left(\mathrm{CF}_{4}\right)$, 1,2-dichlorotetrafluoroethane $\left(\mathrm{C}_{2} \mathrm{Cl}_{2} \mathrm{~F}_{4}\right)$, 
and hexafluoroethane $\left(\mathrm{C}_{2} \mathrm{~F}_{6}\right)$. Among all halon molecules, we have chosen to investigate the excited states of only those which are gaseous under standard conditions of temperature and pressure, primarily because of their importance in atmospheric chemistry. The structures of the studied molecules, alongside their IUPAC names and names according to the special halons' naming convention are shown in Figure 1. To our knowledge, none of the existing molecular sets included any of these molecules. Apart from $\mathrm{CH}_{3} \mathrm{Br}$, whose low-lying excited states are examined in several studies ${ }^{15-17}$, excited states of the other molecules in the set have not yet been studied computationally. Nevertheless, there are numerous experimental studies of the UV spectra of halons (for $\mathrm{CH}_{3} \mathrm{Br}$ ref. ${ }^{18}$ and the references cited herein; for $\mathrm{CBrF}_{3}$ ref. $^{19-21}$ and the references cited herein; for $\mathrm{CF}_{4}$ ref. $^{22,23}$; for $\mathrm{CHCl}_{2} \mathrm{~F}$ ref. ${ }^{24}$; for $\mathrm{CHBrF}_{2}$ ref. $^{21}$; for $\mathrm{CBrClF}_{2}$ ref. $^{21,25,26}$; for $\mathrm{CCl}_{2} \mathrm{~F}_{2}$ ref. $^{24,27}$; for $\mathrm{C}_{2} \mathrm{~F}_{6}$ ref. $^{28}$; for $\mathrm{C}_{2} \mathrm{Cl}_{2} \mathrm{~F}_{4}$ ref. $\left.^{29}\right)$.

This omission may impact the evaluation of computational methods and properties by introducing a negative bias against halogen compounds. We particularly felt that in recent simulations of halo-compounds of atmospheric interest ${ }^{30-34}$, where we could not count on performance evaluation for any of the molecules of interest. Our aim in this study is to start to fill this knowledge gap, first, by proposing a clear set of molecules to be tested; and second, by evaluating them using few popular methods. As a first study, we limited ourselves to the investigation of spin-free vertical excitation energies for the whole series. Important topics as adiabatic excitation energies and spin-orbit coupling will be left for further studies. We probed the performance of two DFT-based quantum chemical methods against equation of motion CCSD (EOM-CCSD) $)^{35-38}$ in the computations of excited states of these systems.

We focus on performance of density functional theory-based multi-reference configuration interaction method (DFT/MRCI) ${ }^{39}$ and TD-DFT in combination with CAM-B3LYP functional $^{40,41}$ in description of excited states of halons. The 240 excited states included in our study comprise a small number of valence excited states in low-energy regions and numerous highly-correlated Rydberg and mixed valence-Rydberg states in high-energy regions converging to the ionization limits.

TD-DFT stands out among other computational methods as one of the most popular due to its good compromise between accuracy and computational cost. Several different flavors of density functionals have been developed, and their performance has been benchmarked 
against high-level ab initio or experimental data so far, probing their performance for a range of excited states, from localized to delocalized ones. ${ }^{42-48}$ One of the main drawbacks of TD-DFT is the description of systems with pronounced multi-reference character of ground state. Moreover, within the usual linear-response and adiabatic approximations, TD-DFT is incapable of reproducing doubly-excited states ${ }^{49}$.

DFT/MRCI overcomes a large number of shortcomings of TD-DFT, because of its multireference character. It is better suited to treat highly-correlated systems, and to capture the excited states of double-excited character. The DFT/MRCI method has been tested for a number of organic molecules ${ }^{39,44,50-52}$ and transition metal complexes $^{53}$, applied for

simulation of UV/vis spectra (e.g. Ref. ${ }^{54-56}$ ), and recently assessed for the computation of spin-orbit coupling in organic molecules ${ }^{57}$. It has been shown that it is able to reproduce excitation energies with errors within few tenths of an eV. However, most of the available benchmark studies of excited states with DFT/MRCI are usually focused on valence excited states of $\mathrm{n} \pi^{*}$ or $\pi \pi^{*}$ character, and do not include higher states characterized by $\sigma$ bond excitations, valence-Rydberg mixing, and Rydberg states.

EOM-CCSD computations of excited states are used as the reference data for the comparison with DFT/MRCI and TD-DFT results. Although it is one of the most reliable methods available for routine excited state calculations, we should mention that, just like TD-DFT, it is not able to describe multi-reference and double excitations. Therefore, concerning these properties, we should take DFT/MRCI as the reference.

\section{METHODS AND COMPUTATIONAL DETAILS}

The ground state molecular structures of the nine molecules in the Halons-9 molecular set have been optimized using Møller-Plesset perturbation theory up to the second order (MP2) $)^{58-61}$ with def2-TZVPP basis set ${ }^{62}$ without symmetry constraints. First ionization potentials of the studied molecules are computed at their optimized structures using coupled cluster with singles and doubles (CCSD) method ${ }^{63-66}$ with the triple-zeta def2-TZVPP basis set, as the difference between the ground state energy of the $N$ and the $N-1$ electron systems. Cartesian coordinates for the optimized structures are given in the Supporting Information (Tables I-IX) and vertical ionization potentials (IPs) are given in Table I. Vertical 
excitation energies of singlet and triplet excited states up to around the ionization limits are computed at the optimized structures at three different levels of theory - EOM-CCSD ${ }^{35-38}$, DFT/MRCI ${ }^{39}$, and linear response TD-DFT. We tested the influence of size of the basis set on excited state energies of $\mathrm{CF}_{4}$ by comparing their values obtained with the def2-TZVPP and quadruple-zeta def2-QZVPP ${ }^{62}$ bases (see Table X in the Supporting Information). The def2-QZVPP basis set decreases the vertical excitation energies of $\mathrm{CF}_{4}$ molecule for $\approx 0.3$ $\mathrm{eV}$ in average. Since we are primarily interested to compare the performances of applied methods, we decided to use smaller def2-TZVPP set in all computations.

Since excited states with pure Rydberg or mixed valence-Rydberg character are expected to occur among the computed excited states, TD-DFT computations are performed employing range-separated (rs) hybrid Coulomb attenuated method CAM-B3LYP functional ${ }^{40,41}$. As it is well-known ${ }^{67}$ generalized gradient approximation (GGA) and hybrid exchange-correlation functionals usually describe accurately local excitations, with an accuracy of few tenths of an $\mathrm{eV}$. However, they significantly underestimate excitation energies of non-local states, such as Rydberg or charge-transfer excitations ${ }^{68}$, with the typical errors of order of an $\mathrm{eV}$. The origin of this problem is already very well-known - it lies in an incorrect description of longrange exchange functionals with GGA and hybrid functionals ${ }^{69-71}$. This problem can be overcome using range-separated functionals, which describe short- and long-range exchange functionals by different terms. One of the most widely used rs functionals, CAM-B3LYP, comprises 0.19 Hartree-Fock(HF)-like and 0.81 Becke 1988 (B88) GGA exchange at short range; the long-range region is represented by 0.65 HF-like plus 0.35 B88 exchange; and the transition between short- and long-range regions is modulated by an error function with parameter $0.33 a_{0}^{-1} \cdot{ }^{40}$ Assessed for the computation of excitation energies of Rydberg and charge transfer states, CAM-B3LYP generally shows good performance. ${ }^{72,73}$

To analyze the delocalization of the excited states obtained on TD-CAM-B3LYP level, we employ the $\Lambda$ diagnostic, introduced by Peach et al. ${ }^{74}$, as a method to quantify the degree of delocalization of states within TD-DFT method. The $\Lambda$ values are computed as sums of spatial overlaps between all occupied and virtual orbital pairs involved in TD-DFT excitations, weighted by their contributions to the specific excited state. These contributions are obtained by solving Casida's equations ${ }^{74}$. The $\Lambda$ value is a dimensionless number which takes value between 0 and 1 ; small $\Lambda$ values signify excitations with strong delocalized characters, whereas large values signify local excitations. All presented TD-DFT computations 
were performed with GAMESS-US code ${ }^{75,76}$.

The basic idea of the combined density functional theory/multi-reference configuration interaction (DFT/MRCI) method is to perform DFT computations of a closed-shell reference ground state, and to use the obtained one-particle Kohn-Sham (KS) orbitals as a basis to subsequently build configuration state functions (CSFs) with short MRCI expansions. ${ }^{39}$ The Hamiltonian matrix elements computed between the CSFs with the same space and same spin parts (diagonal elements) are the most important, because CI-matrix is usually diagonal-dominant. They account for most of dynamic electronic correlation included via DFT. On the other hand, the off-diagonal Hamiltonian matrix elements are computed between CSFs with same space but different spin parts or between CSFs with one-electron or two-electron differences in space parts. In DFT/MRCI off-diagonal matrix elements are scaled using exponential damping function, which depends on the energy difference between diagonal elements of two CSFs. This scaling is introduced to avoid large extent of double counting of electron correlation which is already included via diagonal terms. It also ensures efficient selection of energetically close CSFs, whose interaction gives rise to non-dynamic correlation effects which are not taken into account within DFT. A few semi-empirical screening parameters are employed in the DFT/MRCI Hamiltonan, including parameters used for fitting the exponential damping function for scaling procedure and parameters used for screening of two-electron integrals in diagonal matrix elements.

In our DFT/MRCI computations, the reference configurations were initially generated by single and double excitations of ten active electrons within the active space composed of ten orbitals. We discard the configurations with energies above 1 Hartree. The number of created configurations spans from 946 in the case of $\mathrm{CF}_{4}$ molecule to 10139 in the case of $\mathrm{C}_{2} \mathrm{Cl}_{2} \mathrm{~F}_{4}$ molecule. We employ the original sets of five empirical parameters for singlet and triplet excited states for the DFT/MRCI Hamiltonian ${ }^{39,77}$, which are available in combination with the BH-LYP functional ${ }^{78}$.

The initial DFT calculations are performed with TURBOMOLE program ${ }^{79}$, and the subsequent MRCI computations are done with the MRCI code developed by Grimme and Waletzke $^{39}$ and recently updated by Lyskov and co-workers ${ }^{80}$. The two-electron integrals in DFT/MRCI computations were approximated using the resolution-of-the-identity approximation with auxiliary basis set ${ }^{81}$ from the TURBOMOLE library.

The spin-restricted EOM-CCSD computations and the MP2 geometry optimizations are 
UV Excitations of Halons

performed with Gaussian 09 program $^{82}$.

\section{RESULTS AND DISCUSSION}

\section{A. Vertical excitations}

Computed vertical excitation energies, state assignments, and their $\Lambda$ values are compiled in Table II for the singlet and in Table III for the triplet excited states. Oscillator strengths for the singlet transitions are given as well. For the sake of brevity, we only show the singlet excited states with oscillator strengths larger than 0.001 and only the first 5 triplet excited states for each molecule. Complete Tables including all computed excited states are given in the Supporting Information (Table XI and Table XII).

The assignment of the excited states computed with the applied methods is done by analyzing the nature of orbitals involved in the main excitations contributing to each particular state. The states computed with different methods were correlated according to their determined types and weights of main configurations. In the case of several high-lying multiconfigurational states, correlation of the excited states computed with EOM-CCSD and the DFT-based methods according to these criteria is non-trivial due to a pronounced configuration mixing within EOM-CCSD, which is not particularly well reproduced with DFT/MRCI and TD-CAM-B3LYP methods. In these cases, the comparison of the oscillator strength values and the symmetry representation of the states were also helpful for the assignment. Weights of the main configurations obtained with EOM-CCSD are given in the tables in the cases where it was possible to determine them. For numerous states, however, arising from the excitations between mixed orbitals (represented as linear combinations of different

orbital types, for instance $\mathrm{n} \sigma^{*}$ and Rydberg orbitals), the configuration weights are not computed.

According to the DFT/MRCI results, all computed states are single-reference and singlyexcited, with negligible contributions of doubly-excited configurations (less than $5 \%$ ). This implies that all states obtained with DFT/MRCI could in principle be also computed with EOM-CCSD and TD-DFT methods, which are unsuited to capture states with predominantly multi-reference and doubly-excited character.

Based on the assignment, the benchmark set of 240 excited states was split into 64 localized 
valence states, 91 Rydberg states, and 85 states of mixed valence-Rydberg character; and into 110 singlet and 130 triplet states. The correlation graphs between the TD-CAM-B3LYP and DFT/MRCI vertical excitation energies with the corresponding EOM-CCSD values are shown in Figure 2, separately for singlet and triplet excited states. Some of the features of the computed states are noticeable from first sight. Both DFT/MRCI and TD-CAMB3LYP underestimate excitation energies of all computed states. DFT/MRCI excitation energies lie in between EOM-CCSD and TD-CAM-B3LYP values, significantly closer to the EOM-CCSD values. The standard deviation of DFT/MRCI vertical excitation energies from the corresponding EOM-CCSD values is 0.49 for singlet and 0.51 for triplet states, whereas for TD-CAM-B3LYP these values are 0.72 and 0.79 respectively. Both methods describe slightly better singlet than triplet states.

For most of molecules, several pure valence excited singlet and triplet states occur in the low energy region. They are usually of $n \sigma^{*}$ type, but there are also several states of $\sigma \sigma^{*}$ or mixed $\mathrm{n} \sigma^{*}-\sigma \sigma^{*}$ type. These states emerge upon excitations from the highest HOMO orbitals of non-bonding or $\sigma$ type to the lowest-lying LUMO and LUMO+1 orbitals. In these cases, the lowest-lying LUMO orbitals are anti-bonding $\sigma^{*}(\mathrm{C}-\mathrm{Br})$ or $\sigma^{*}(\mathrm{C}-\mathrm{Cl})$. Exceptions to this behavior are $\mathrm{CF}_{4}$ and $\mathrm{C}_{2} \mathrm{~F}_{6}$ molecules, where the LUMO orbitals are Rydberg ones. This induces the lowest-lying excited states of $\mathrm{CF}_{4}$ and $\mathrm{C}_{2} \mathrm{~F}_{6}$ to be Rydberg. Since the energies of the $\sigma^{*}(\mathrm{C}-\mathrm{F})$ orbitals are higher than the energies of the Rydberg orbitals, states of pure $\mathrm{n} \sigma^{*}$ and $\sigma \sigma^{*}$ character do not occur, whereas states with mixed Rydberg and n $\sigma^{*}$ configurations occur only in the upper parts of their spectra, contrary to the other molecules in the set (Table XI and Table XII, Supporting Information). In all cases, the medium part of the excited state spectrum is characterized by numerous multiconfigurational excited states featured by considerable valence-Rydberg mixing.

The highest excited states are mostly purely Rydberg states. In several cases, few delocalized states of $n \sigma^{*}$ character appear in the upper part of the spectrum. These states arise from excitations from the compact non-bonding orbitals of fluorine to the $\sigma^{*}(\mathrm{C}-\mathrm{Br})$ or $\sigma^{*}(\mathrm{C}-\mathrm{Cl})$ orbitals, which have a small spatial overlap.

This pattern occurring in the excited states spectrum could be explained by energy ordering of molecular orbitals, common to all molecules, $\sigma(\mathrm{C}-\mathrm{F})<\sigma(\mathrm{C}-\mathrm{Cl})<\sigma(\mathrm{C}-\mathrm{Br})<\mathrm{n}(\mathrm{F})<$ $\mathrm{n}(\mathrm{Cl})<\mathrm{n}(\mathrm{Br})<\sigma^{*}(\mathrm{C}-\mathrm{Br})<\sigma^{*}(\mathrm{C}-\mathrm{Cl})<$ Rydberg $<\sigma(\mathrm{C}-\mathrm{F})$. The compositions of the lowest Rydberg orbitals varies, but in most of cases they are represented as linear combinations 
of several atomic orbitals rather than as a pure one. Based on this ordering of orbitals, it could be predicted that in the molecules containing $\mathrm{C}-\mathrm{Cl}$ and $\mathrm{C}-\mathrm{Br}$ bonds, the lowest-lying excited states would be of $\sigma^{*}$ character, while in molecules containing only $\mathrm{C}-\mathrm{F}$ and $\mathrm{C}-\mathrm{C}$ bonds, the lowest lying excited states would be Rydberg states.

\section{B. TD-DFT results}

A summary of statistical evaluation, including mean errors (ME), mean absolute errors (MAE), standard deviations (SD), maximum and minimum errors for TD-CAM-B3LYP and DFT/MRCI results with respect to EOM-CCSD results is given in Table IV and Figure 3, separately for valence, mixed valence-Rydberg, Rydberg, and all studies states. Comparing TD-CAM-B3LYP vertical excitation energies with the corresponding EOM-CCSD values, it could be noticed that among all the excited states, valence states are described the best on TD-CAM-B3LYP level. Their MAE from the EOM-CCSD results is $0.58 \mathrm{eV}$. Analyzing the valence state compositions, we find that the lowest-lying valence states, arising upon excitations from the highest occupied non-bonding orbitals to the LUMO orbital, are described fairly well. The MAE of their vertical excitation energies from EOM-CCSD values is 0.38 $\mathrm{eV}$ for singlet (18 states) and $0.48 \mathrm{eV}$ for triplet (20) excited states. These states are mostly $\mathrm{n} \sigma^{*}$ states represented mainly by a single configuration (Table II, Table III, and Table XI, Table XII in the Supporting Information).

The description of the higher excited states deteriorates in the upper region of the spectrum. In several cases, the ordering of the states obtained with TD-CAM-B3LYP differs from that obtained with EOM-CCSD. The excitation energies of higher valence, mixed valence-Rydberg, and Rydberg states are severely underestimated. The high-energy n $\sigma^{*}$ valence excited states occurring in $\mathrm{CCl}_{2} \mathrm{~F}_{2}, \mathrm{CBrClF}_{2}$, and $\mathrm{C}_{2} \mathrm{Cl}_{2} \mathrm{~F}_{4}$ are placed significantly lower compared to the EOM-CCSD results (their maximum deviation is $1.1 \mathrm{eV}$ ). The most dominant configurations contributing to these states involve transitions from the highest $\mathrm{HOMO}$ orbitals to LUMO+1 orbitals, which is the $\sigma^{*}\left(\mathrm{C}-\mathrm{Cl}\right.$ ) orbital (in the cases of $\mathrm{CCl}_{2} \mathrm{~F}_{2}$ and $\mathrm{C}_{2} \mathrm{Cl}_{2} \mathrm{~F}_{4}$ this is the higher $\sigma^{*}(\mathrm{C}-\mathrm{Cl})$ orbital).

The excitation energies of the pure Rydberg states are also systematically underestimated compared to the EOM-CCSD results, with MAE of $0.76 \mathrm{eV}$ and maximum error of $1.20 \mathrm{eV}$. We employ the $\Lambda$ diagnostic to analyze the correlation between the degree of delocalization 
of the states with dominant Rydberg character and the errors of their excitation energies. The obtained $\Lambda$ values of the studied Rydberg states are in the range between 0.2 and 0.65 (Figure 1 in the Supporting Information). Analyzing the deviations of TD-CAM-B3LYP excitation energies of Rydberg states against the corresponding $\Lambda$ values for Rydberg excited states, it could be noticed that in the cases of three molecules $\left(\mathrm{C}_{2} \mathrm{~F}_{6}, \mathrm{CBrF}_{3}, \mathrm{C}_{2} \mathrm{Cl}_{2} \mathrm{~F}_{4}\right)$ there is no correlation between excitation energy errors and $\Lambda$ values. In the majority of cases, however, the deviations of the excitation energies are larger in the cases of more delocalized states, which are characterized by smaller $\Lambda$ values. The excitation energy errors for most of molecules slowly reduce upon increasing of $\Lambda$ values (Figure 1 and Table XI in the Supporting Information), but they still remain large (close to $1 \mathrm{eV}$ ) even for Rydberg states with relatively large $\Lambda$ values.

A tendency of improvement of the excitation energies for the states with larger $\Lambda$ values could indicate that CAM-B3LYP functional does not describe correctly the long-range behavior of the exchange functional and that additional asymptotic correction of the functional is necessary. This behavior of CAM-B3LYP functional has already been observed before in several different benchmark studies (e.g. Ref. ${ }^{74}$ ). However, in the case of halons, the MAE of the Rydberg states' excitation energies $(0.76 \mathrm{eV})$ is close to the one of the valence states $(0.56 \mathrm{eV})$, and it is smaller than the corresponding value of the mixed valence-Rydberg states $(1.06 \mathrm{eV})$. This indicates that the eventual incorrect description of the long-range behavior of the exchange functional with TD-CAM-B3LYP is not the most important source of the errors of excitation energies of halons.

The excitation energies of the states with mixed valence-Rydberg character are also severely underestimated on TD-CAM-B3LYP level. The MAE of the excitation energies compared to EOM-CCSD results is $1.06 \mathrm{eV}$, whereas the maximum deviation is $1.33 \mathrm{eV}$. Such large excitation energy errors are due to underestimation of the excitation energies of Rydberg orbitals, but also seem to be correlated to the pronounced multiconfigurational character of these states.

The $\Lambda$ values of the states with mixed valence-Rydberg character are spread in similar region as pure Rydberg states (Figure 1, Supporting Information), from 0.2 to 0.7, but their mean $\Lambda$ value (0.73) is larger than the mean $\Lambda$ value for pure Rydberg states (0.65). Also, their mean deviation from the EOM-CCSD results $(1.06 \mathrm{eV})$ is larger than one for Rydberg states $(0.76 \mathrm{eV})$. The low-lying valence states have $\Lambda$ values concentrated in the region from 0.55 
to 0.65 (Figure 1, Supporting Information).

One of the interesting features occurring in the case of $\mathrm{CCl}_{2} \mathrm{~F}_{2}$ and $\mathrm{CBrF}_{3}$ molecules are the several $\mathrm{n} \sigma^{*}$ excited states arising from excitation from non-bonding orbital of fluorine to antibonding $\sigma^{*}(\mathrm{C}-\mathrm{Cl})$ or $\sigma^{*}(\mathrm{C}-\mathrm{Br})$ orbitals, which could be classified as delocalized. These states are featured by very small $\Lambda$ values, since the overlaps between localized non-bonding fluorine orbitals and antibonding $\sigma^{*}$ orbitals are small in these molecules. According to EOM-CCSD results, these states occur in the highest part of the spectrum, above Rydberg states. TD-CAM-B3LYP underestimates significantly the excitation energies of these states.

TD-CAM-B3LYP performs the best in the case of the excited states of $\mathrm{CH}_{3} \mathrm{Br}$ molecule (MAE is $0.65 \mathrm{eV}$ ), whereas it gives the largest errors for the excited states of $\mathrm{CF}_{4}(1.03 \mathrm{eV})$ and $\mathrm{C}_{2} \mathrm{~F}_{6}(0.93 \mathrm{eV})$, which are mainly Rydberg states.

Considering all states, TD-CAM-B3LYP systematically underestimates the excitation energies in the Halons-9 set, with a MAE of $0.82 \mathrm{eV}$ (Table IV). This error is astonishingly large given the usual $0.3 \mathrm{eV}$ MAE usually observed in benchmarks based on organic molecular sets ${ }^{13}$ which are biased towards low-lying $n \pi^{*}$ and $\pi \pi^{*}$ states. This deterioration of the TD-DFT energies for high excitations was recognized long ago by Casida et al. ${ }^{68}$, who traced it back to a wrong asymptotic behaviour of the exchange-correlation (XC) potential. Since then, diverse schemes have been proposed to fix the asymptotic behavior, ${ }^{68,83,84}$ although none of them has yet been adopted as standard in TD-DFT calculations. One of the features of this problem with the $\mathrm{XC}$ potential is the strong underestimation of $-E_{\text {HOMO }}$ (the negative of the HOMO energy), lying much lower than the true IP; with a consequent collapse of the higher excited states in the region between $-E_{\text {HOMO }}$ and the IP. ${ }^{49}$ It goes beyond the scope of this work to attempt to correct this feature here, as our focus is at the performance of standards methods. However, given the strong deviation between $-E_{\text {Hомо }}$ and the IP at CAM-B3LYP level (see Table I), we note that an adequate treatment of the $\mathrm{XC}$ potential may help to improve the TD-DFT description of the excited state of halons.

Finally, the obtained oscillator strengths agree well with the EOM-CCSD values. The largest discrepancies are found in the cases of several multiconfigurational states, which are poorly described with TD-CAM-B3LYP. 


\section{DFT/MRCI results}

The DFT/MRCI excitation energies for all studied states are also underestimated compared to the EOM-CCSD values, but they provide much better description of the excited states; the DFT/MRCI excitation energies lie much closer to the EOM-CCSD values. The ordering of the excited states according to DFT/MRCI coincides with the one predicted by EOM-CCSD.

Similarly as in the case of TD-CAM-B3LYP, the description of the lowest-lying valence states, which arise upon excitations from the several highest occupied orbitals to the LUMO orbital, is fairly good. However, the description of the higher excited states deteriorates. The deviations of the excitation energies from the corresponding EOM-CCSD values are larger for the higher valence, Rydberg, and mixed valence-Rydberg states. The largest deviations occur in the case of the higher valence states, where the maximum deviation from the EOM-CCSD results is $1.02 \mathrm{eV}$ (Table IV, Figure 3). These states mainly arise due to excitations to LUMO+1 orbitals. The MAE of all valence states (including those formed by excitations to LUMO and to LUMO+1 orbitals) is $0.44 \mathrm{eV}$, reflecting an overall satisfying performance of DFT/MRCI for these states.

The medium and upper parts of the excited state spectra of halons are mainly featured by states with strong valence-Rydberg mixing. As a CI-based method, it is expected that DFT/MRCI should be flexible enough to treat properly these states. In principle, the DFT/MRCI description of these states is more reliable compared to TD-CAM-B3LYP, but the obtained deviations from EOM-CCSD are still significant; the MAE of the states with mixed valence-Rydberg character is $0.66 \mathrm{eV}$ and their maximum error is $0.80 \mathrm{eV}$. The description of Rydberg states, on the other hand, is significantly improved compared to TDCAM-B3LYP results; the MAE of the Rydberg states is $0.47 \mathrm{eV}$ and the maximum error is $0.80 \mathrm{eV}$. Insufficiently good performances of DFT/MRCI in the cases of multiconfigurational highly-correlated excited states of several systems have already been noted before. ${ }^{53,80}$ DFT/MRCI describes slightly better singlet than triplet excited states. The mean average error of the triplet states $(0.53 \mathrm{eV})$ is a little bit larger compared to the error of the singlet states $(0.47 \mathrm{eV})$. The trend of inferior description of triplet states, common to all DFT-based methods, has already been noticed in the case of some other benchmarking sets ${ }^{44,77}$. DFT/MRCI describes very well excited states of $\mathrm{CF}_{4}, \mathrm{C}_{2} \mathrm{~F}_{6}, \mathrm{CF} 3 \mathrm{Br}$, and $\mathrm{CH}_{3} \mathrm{Br}$ molecules, 
whereas the largest deviations are found in the cases of $\mathrm{CCl}_{2} \mathrm{~F}_{2}, \mathrm{C}_{2} \mathrm{Cl}_{2} \mathrm{~F}_{4}$, and $\mathrm{CBrClF}_{2}$ molecules. The bad performance in these cases is due to occurrence of the highly correlated multiconfigurational valence and valence-Rydberg states, for which the performance of DFT/MRCI is not good enough. Globally, considering all states the MAE if 0. $53 \mathrm{eV}$. Most of the computed DFT/MRCI oscillator strengths are in the range of the corresponding EOM-CCSD values. Only in a number of high-lying Rydberg and valence-Rydberg states with strong configuration mixing, which are not described very well with DFT/MRCI, there are sometimes discrepancies between DFT/MRCI and EOM-CCSD oscillator strengths.

In the last several years a lot of effort is put into development of a new method which unites advantages of multireference methods and DFT, the time-dependent multiconfigurational short-range density functional theory (TD-MC-srDFT) method $^{85}$. It consists in combining a long-range multi-configuration self-consistent field (MCSCF) treatment with an adiabatic short-range density-functional (DFT) description extended for computation of excited states in time-dependent regime. Benchmarked on a set of small organic molecules ${ }^{86}$ and nucleobases ${ }^{87}$, the TD-MC-sdDFT method in the CAS-srPBE variant generally showed good performance. It is suitable for computation of doubly-excited states and excited states in molecules with multirefence ground-state functions. However, the TD-MC-srDFT method is not reliable for description of states which display Rydberg-valence mixing ${ }^{86,87}$. In their cases large errors of excitation energies are observed, and it is found that they are dependent on a chosen value of range-separation parameter ${ }^{86,87}$. However, it is expected that different variants of TD-MX-srDFT method obtained combining other functionals and multireference methods, which are not tested yet, might provide better results for mixed valence-Rydberg states.

\section{Comparison with the experimental data}

The computed vertical excitation energies and the positions of band peaks of available experimental UV photoabsorption spectra are compiled in Table V. However, comparison of the computed excitation energies with the peak positions is problematic due to influences of spin-orbit and adiabatic effects which were not taken into account in computations of excitation energies. It is expected that spin-orbit coupling would be particularly intensive in the case of molecules which contain bromine, which is a relatively heavy element. 
Man et al. ${ }^{19}$ computed absorption spectrum of bromomethane using time-dependent approach to resonance Raman scattering. They deconvoluted the A-band in gas-phase absorption spectra into three transitions: ${ }^{3} Q_{1}$ at $45260 \mathrm{~cm}^{-1}(5.61 \mathrm{eV}),{ }^{3} Q_{0}$ at $48272 \mathrm{~cm}^{-1}(5.98$ $\mathrm{eV})$, and ${ }^{1} Q_{1}$ at $51372 \mathrm{~cm}^{-1}(6.37 \mathrm{eV})$. The positions of ${ }^{3} Q_{0}$ and ${ }^{1} Q_{1}$ bands are close to the values of EOM-CCSD excitation energies of the first triplet $(6.01 \mathrm{eV})$ and singlet $(6.64 \mathrm{eV})$ states, respectively (Table V).

In the cases of other molecules the recorded UV spectra are not resolved into spin components. In several cases the positions of band peaks are closer to $S_{1}$ and in some cases to $T_{1}$ excitation energies. In the cases of highly symmetric molecules $\left(\mathrm{CF}_{4}, \mathrm{C}_{2} \mathrm{~F}_{6}, \mathrm{CCl}_{2} \mathrm{~F}_{2}, \mathrm{CBrF}_{3}\right.$, $\mathrm{CH}_{3} \mathrm{Br}$ ) with doubly-degenerate $S_{1}$ and $T_{1}$ states, comparison the reference EOM-CCSD results with the experimental ones is even more difficult, because of vibronic interactions which influence the excitation energies of these states. For these reasons, we cannot draw any conclusion about validity of the reference EOM-CCSD method. A more comperhensive study of excited states with inclusion of spin-orbit and adiabatic effects is necessary in order to compare ab initio and experimental data.

\section{CONCLUSIONS}

Given the complete lack of excited state information about halo-organic compounds in excited state benchmarks, we have introduced a new molecular set tailored for evaluation of molecular properties of such systems. The Halons-9 set is composed of nine small gaseous halons, including $\mathrm{F}, \mathrm{Cl}$, and $\mathrm{Br}$ elements. Vertical excitation energies were assessed with two DFT-based methods, TD-DFT with CAM-B3LYP functional and DFT/MRCI methods, taking CCSD as the reference method.

The present benchmark shows that the evaluated methods only provide semi-quantitative results, with errors in the order of several tenths of an eV. Overall, we observed a superior performance of multi-reference DFT/MRCI method in the calculation of the excited states of halons (MAE is $0.53 \mathrm{eV}$ ), compared to TD-CAM-B3LYP method (MAE is $0.82 \mathrm{eV}$ ) (Table IV, Figure 3).

TD-CAM-B3LYP method performs the best for the lowest-lying valence states represented mainly by single configurations (MAE is $0.56 \mathrm{eV}$ ). In the case of higher valence states, states 
with pronounced valence-Rydberg mixing, and Rydberg states, TD-CAM-B3LYP vertical excitation energies are significantly underestimated compared to EOM-CCSD values. The description of the mixed valence-Rydberg states with TD-CAM-B3LYP is the most challenging; the MAE of the mixed valence-Rydberg states is the largest $(1.06 \mathrm{eV})$, whereas the MAE of the pure Rydberg states $(0.76 \mathrm{eV})$ is considerably larger than the one of the valence states $(0.56 \mathrm{eV})$.

On the other hand, DFT/MRCI method performs reasonably well for most of the studied states. It gives much more reliable description of states with pronounced multiconfigurational character, which appear in the middle and upper parts of the spectrum, compared to TD-CAM-B3LYP. The largest deviations from the EOM-CCSD values are observed in the case of higher-lying multiconfigurational mixed valence-Rydberg states.

Contrary to expectations based on previous numerous benchmarking studies, where valence excited states are usually described with errors around 0.2 to $0.3 \mathrm{eV}$ with TD-DFT or DFT/MRCI, the valence excited states of Halons-9 molecular set are described only semiquantitatively with both methods (the MAE of valence states' excitation energies obtained with TD-CAM-B3LYP and DFT/MRCI are 0.58 and $0.44 \mathrm{eV}$, respectively). The reason for this is in the fact that most of benchmark studies focus on organic molecules with low-lying $n \pi^{*}$ or $\pi \pi^{*}$ excited states. By construction, the excited states in the Halons-9 molecular set ( $n \sigma^{*}, \sigma \sigma^{*}, \mathrm{n}$-Rydbergs, and mixed valence-Rydbergs) are much higher. In this spectral region, the effects of wrong asymptotic behaviour of the $\mathrm{XC}$ potential are much more pronounced, leading to a deterioration of the results.

Oscillator strengths for most of the transitions are described satisfactorily with both methods. The exceptions are strongly-correlated multiconfigurational states, where, in the cases of several states, oscillator strengths strongly deviate from the EOM-CCSD values.

All molecules in the Halons-9 set are featured by electronic transitions in far- and extreme UV-region, implying that these transitions cannot be photoactivated close to the Earth's surface, although they may play a role in the photochemistry of the high atmosphere.

We were not able to evaluate the validity of the EOM-CCSD method, which was used as the reference, because of the UV excitation of most of molecules in the Halons-9 set should be strongly affected by spin-orbit couplings. For this reason, we limited our analysis to computational methods. The effect of spin-orbit coupling and adiabatic effects on excited state of Halons-9 set will be investigated in our future work. 


\section{ACKNOWLEDGMENTS}

This Project was funded by the Deanship of Scientific Research (DSR) King Abdulaziz University, Jeddah, under grant no.43-130-35-RG. The authors, therefore, acknowledge with thanks DSR support for Scientific Research. Also, the authors appreciate the kind cooperation provided by the Deanship of Scientific Research (DSR), King Abdulaziz University. MB and LS thank the support of the A*MIDEX grant (no. ANR-11-IDEX-0001-02) and of the project Equip@Meso (ANR-10-EQPX-29-01), both funded by the French Government "Investissements d'Avenir" program. They are also in debt to Prof. Christel Marian, who kindly provided the latest version of the DFT/MRCI program. LS also acknowledges Miquel Huix-Rottlant for help with the GAMESS code.

\section{REFERENCES}

${ }^{1}$ E. Runge and E. K. U. Gross, "Density-functional theory for time-dependent systems," Phys. Rev. Lett. 52, 997-1000 (1984).

${ }^{2}$ M. E. Casida, "Time-dependent density functional response theory for molecules," in $R e$ cent Advances in Density Functional Methods (WORLD SCIENTIFIC, 2011) pp. 155-192.

${ }^{3}$ K. Andersson, P. Malmqvist, and B. O. Roos, "Second-order perturbation theory with a complete active space self-consistent field reference function," The Journal of Chemical Physics 96, 1218-1226 (1992).

${ }^{4}$ R. J. Buenker and S. D. Peyerimhoff, "CI method for the study of general molecular potentials," Theoretica chimica acta 12, 183-199 (1968).

${ }^{5}$ M. Schreiber, M. R. Silva-Junior, S. P. A. Sauer, and W. Thiel, "Benchmarks for electronically excited states: CASPT2, CC2, CCSD, and CC3," The Journal of Chemical Physics 128, 134110 (2008).

${ }^{6}$ M. R. Silva-Junior, M. Schreiber, S. P. A. Sauer, and W. Thiel, "Benchmarks for electronically excited states: Time-dependent density functional theory and density functional theory based multireference configuration interaction," The Journal of Chemical Physics 129, 104103 (2008). 
UV Excitations of Halons

${ }^{7}$ S. P. A. Sauer, M. Schreiber, M. R. Silva-Junior, and W. Thiel, "Benchmarks for electronically excited states: A comparison of noniterative and iterative triples corrections in linear response coupled cluster methods: CCSDR(3) versus CC3," Journal of Chemical Theory and Computation 5, 555-564 (2009).

${ }^{8}$ M. R. Silva-Junior, M. Schreiber, S. P. A. Sauer, and W. Thiel, "Benchmarks of electronically excited states: Basis set effects on CASPT2 results," The Journal of Chemical Physics 133, 174318 (2010).

${ }^{9}$ M. Parac and S. Grimme, "Comparison of multireference Møller-Plesset theory and timedependent methods for the calculation of vertical excitation energies of molecules," The Journal of Physical Chemistry A 106, 6844-6850 (2002).

${ }^{10}$ M. Dierksen and S. Grimme, "Density functional calculations of the vibronic structure of electronic absorption spectra," The Journal of Chemical Physics 120, 3544-3554 (2004).

${ }^{11}$ L. Goerigk, J. Moellmann, and S. Grimme, "Computation of accurate excitation energies for large organic molecules with double-hybrid density functionals," Phys. Chem. Chem. Phys. 11, 4611-4620 (2009).

${ }^{12}$ M. Parac and S. Grimme, "A TDDFT study of the lowest excitation energies of polycyclic aromatic hydrocarbons," Chemical Physics 292, 11 - 21 (2003).

${ }^{13}$ S. S. Leang, F. Zahariev, and M. S. Gordon, "Benchmarking the performance of timedependent density functional methods," The Journal of Chemical Physics 136, 104101 (2012).

${ }^{14}$ N. O. C. Winter, N. K. Graf, S. Leutwyler, and C. Hattig, "Benchmarks for 0-0 transitions of aromatic organic molecules: DFT/B3LYP, ADC(2), CC2, SOS-CC2 and SCS-CC2 compared to high-resolution gas-phase data," Phys. Chem. Chem. Phys. 15, 6623-6630 (2013).

${ }^{15}$ C. Escure, T. Leininger, and B. Lepetit, "Ab initio study of methyl-bromide photodissociation in the A band," The Journal of Chemical Physics 130, 244305 (2009).

${ }^{16} \mathrm{C}$. Escure, T. Leininger, and B. Lepetit, "Ab initio study of valence and Rydberg states of CH3Br," The Journal of Chemical Physics 130, 244306 (2009).

${ }^{17} \mathrm{~A}$. Yu, K. Efil, R. Yang, and Q. Hu, "A comprehensive theoretical investigation of the molecular properties of methyl bromide (CH3Br)," Zeitschrift für Naturforschung A 70, 1025-1030 (2015). 
${ }^{18}$ R. Locht, B. Leyh, H. Jochims, and H. Baumgärtel, "The vacuum UV photoabsorption spectrum of methyl bromide (CH3Br) and its perdeuterated isotopomer CD3Br: a Rydberg series analysis," Chemical Physics 317, 73 - 86 (2005).

${ }^{19}$ S. Man, W. M. Kwok, D. L. Phillips, and A. E. Johnson, "Short-time photodissociation dynamics of A-band and B-band bromoiodomethane in solution: An examination of bond selective electronic excitation," The Journal of Chemical Physics 105, 5842-5857 (1996).

${ }^{20}$ F. Bernard, M. R. McGillen, E. L. Fleming, C. H. Jackman, and J. B. Burkholder, "CBrF3 (Halon-1301): UV absorption spectrum between 210 and $320 \mathrm{~K}$, atmospheric lifetime, and ozone depletion potential," Journal of Photochemistry and Photobiology A: Chemistry 306, 13-20 (2015).

${ }^{21}$ V. L. Orkin and E. E. Kasimovskaya, "Ultraviolet absorption spectra of some Br-containing haloalkanes," Journal of Atmospheric Chemistry 21, 1-11 (1995).

${ }^{22}$ R. Chim, R. Kennedy, and R. Tuckett, "The vacuum-UV absorption spectrum of SF5CF3; implications for its lifetime in the earths atmosphere," Chemical Physics Letters 367, 697 -703 (2003).

${ }^{23}$ P. Hatherly and A. Flaxman, "On the absolute absorption cross-section of SF5CF3," Chemical Physics Letters 380, 512 - 515 (2003).

${ }^{24}$ P. C. Simon, D. Gillotay, N. Vanlaethem-Meuree, and J. Wisemberg, "Ultraviolet absorption cross-sections of chloro and chlorofluoro-methanes at stratospheric temperatures," Journal of Atmospheric Chemistry 7, 107-135 (1988).

${ }^{25}$ D. K. Papanastasiou, N. R. Carlon, J. A. Neuman, E. L. Fleming, C. H. Jackman, and J. B. Burkholder, "Revised UV absorption spectra, ozone depletion potentials, and global warming potentials for the ozone-depleting substances CF2Br2, CF2ClBr, and CF2BrCF2Br," Geophysical Research Letters 40, 464-469 (2013).

${ }^{26}$ L. T. Molina, M. J. Molina, and F. S. Rowland, "Ultraviolet absorption cross sections of several brominated methanes and ethanes of atmospheric interest," The Journal of Physical Chemistry 86, 2672-2676 (1982).

${ }^{27}$ P. L. Vieira, S. Eden, P. Kendall, N. Mason, and S. Hoffmann, "VUV photo-absorption cross-section for CCl2F2," Chemical Physics Letters 364, 535 - 541 (2002).

${ }^{28}$ P. Sauvageau, J. Doucet, R. Gilbert, and C. Sandorfy, "Vacuum ultraviolet and photoelectron spectra of fluoroethanes," The Journal of Chemical Physics 61, 391-395 (1974). 
${ }^{29}$ P. C. Simon, D. Gillotay, N. Vanlaethem-Meuree, and J. Wisemberg, "Temperature dependence of ultraviolet absorption cross-sections of chlorofluoroethanes," Annales Geophysicae 6, 239-248 (1988).

${ }^{30}$ L. Stojanović, G. P. Rodrigues, S. G. Aziz, R. H. Hilal, and M. Barbatti, "Photochemistry of methyl hypobromite (CH3OBr): excited states and photoabsorption spectrum," RSC Adv. 5, 97003-97015 (2015).

${ }^{31}$ G. P. Rodrigues, E. Ventura, S. A. do Monte, and M. Barbatti, "Photochemical deactivation process of HCFC-133a (C2H2F3Cl): A nonadiabatic dynamics study," The Journal of Physical Chemistry A 118, 12041-12049 (2014).

${ }^{32}$ G. P. Rodrigues, J. R. Lucena, E. Ventura, and S. A. do Monte, "Accurate calculation of the ionization energies of the chlorine lone pairs in 1,1,1-trifluoro-2-chloroethane (HCFC133a)," Journal of Molecular Modeling 20, 1-8 (2014).

${ }^{33}$ G. P. Rodrigues, E. Ventura, S. Andrade do Monte, and M. Barbatti, "UV-photoexcitation and ultrafast dynamics of $\mathrm{HCFC}-132 \mathrm{~b}(\mathrm{CF} 2 \mathrm{ClCH} 2 \mathrm{Cl})$," Journal of Computational Chemistry $\mathbf{3 7}, 675-683$ (2016).

${ }^{34}$ V. C. de Medeiros, R. B. de Andrade, E. F. V. Leitāo, E. Ventura, G. F. Bauerfeldt, M. Barbatti, and S. A. do Monte, "Photochemistry of $\mathrm{CH} 3 \mathrm{Cl}$ : Dissociation and CHCl hydrogen bond formation," Journal of the American Chemical Society 138, 272-280 (2016).

${ }^{35}$ H. Koch and P. Jørgensen, "Coupled cluster response functions," The Journal of Chemical Physics 93, 3333-3344 (1990).

${ }^{36}$ J. F. Stanton and R. J. Bartlett, "The equation of motion coupled-cluster method. a systematic biorthogonal approach to molecular excitation energies, transition probabilities, and excited state properties," The Journal of Chemical Physics 98, 7029-7039 (1993).

${ }^{37}$ H. Koch, R. Kobayashi, A. Sanchez de Merás, and P. Jørgensen, "Calculation of sizeintensive transition moments from the coupled cluster singles and doubles linear response function," The Journal of Chemical Physics 100, 4393-4400 (1994).

${ }^{38}$ M. Kállay and J. Gauss, "Calculation of excited-state properties using general coupledcluster and configuration-interaction models," The Journal of Chemical Physics 121, 92579269 (2004).

${ }^{39} \mathrm{~S}$. Grimme and M. Waletzke, "A combination of Kohn-Sham density functional theory and multi-reference configuration interaction methods," The Journal of Chemical Physics 111, 5645-5655 (1999). 
${ }^{40}$ T. Yanai, D. P. Tew, and N. C. Handy, "A new hybrid exchange-correlation functional using the coulomb-attenuating method (CAM-B3LYP)," Chemical Physics Letters 393, $51-57$ (2004).

${ }^{41}$ Y. Tawada, T. Tsuneda, S. Yanagisawa, T. Yanai, and K. Hirao, "A long-range-corrected time-dependent density functional theory," The Journal of Chemical Physics 120, 84258433 (2004).

${ }^{42}$ J. Fabian, L. Diaz, G. Seifert, and T. Niehaus, "Calculation of excitation energies of organic chromophores: a critical evaluation," Journal of Molecular Structure: THEOCHEM 594, 41 - 53 (2002).

${ }^{43}$ D. Jacquemin, E. A. Perpéte, G. Scalmani, M. J. Frisch, R. Kobayashi, and C. Adamo, "Assessment of the efficiency of long-range corrected functionals for some properties of large compounds," The Journal of Chemical Physics 126 (2007).

${ }^{44}$ M. R. Silva-Junior, M. Schreiber, S. P. A. Sauer, and W. Thiel, "Benchmarks for electronically excited states: Time-dependent density functional theory and density functional theory based multireference configuration interaction," The Journal of Chemical Physics 129, 104103 (2008).

${ }^{45}$ D. Jacquemin, E. A. Perpéte, G. Scalmani, M. J. Frisch, R. Kobayashi, and C. Adamo, "Assessment of the efficiency of long-range corrected functionals for some properties of large compounds," The Journal of Chemical Physics 126, 144105 (2007).

${ }^{46}$ D. Jacquemin, V. Wathelet, E. A. Perpéte, and C. Adamo, "Extensive TD-DFT benchmark: Singlet-excited states of organic molecules," Journal of Chemical Theory and Computation 5, 2420-2435 (2009).

${ }^{47}$ K. A. Nguyen, P. N. Day, and R. Pachter, "The performance and relationship among range-separated schemes for density functional theory," The Journal of Chemical Physics 135, 074109 (2011).

${ }^{48}$ A. Charaf-Eddin, A. Planchat, B. Mennucci, C. Adamo, and D. Jacquemin, "Choosing a functional for computing absorption and fluorescence band shapes with TD-DFT," Journal of Chemical Theory and Computation 9, 2749-2760 (2013).

${ }^{49}$ M. Casida and M. Huix-Rotllant, "Progress in time-dependent density-functional theory," Annual Review of Physical Chemistry 63, 287-323 (2012).

${ }^{50}$ A. B. J. Parusel and S. Grimme, "DFT/MRCI calculations on the excited states of porphyrin, hydroporphyrins, tetrazaporphyrins and metalloporphyrins," Journal of Por- 
phyrins and Phthalocyanines 05, 225-232 (2001).

${ }^{51}$ C. M. Marian and N. Gilka, "Performance of the density functional theory/multireference configuration interaction method on electronic excitation of extended $\pi$-systems," Journal of Chemical Theory and Computation 4, 1501-1515 (2008).

${ }^{52}$ S. S. Leang, F. Zahariev, and M. S. Gordon, "Benchmarking the performance of timedependent density functional methods," The Journal of Chemical Physics 136, 104101 (2012).

${ }^{53}$ D. Escudero and W. Thiel, "Assessing the density functional theory-based multireference configuration interaction (DFT/MRCI) method for transition metal complexes," The Journal of Chemical Physics 140, 194105 (2014).

${ }^{54}$ A. B. J. Parusel and S. Grimme, "DFT/MRCI calculations on the excited states of porphyrin, hydroporphyrins, tetrazaporphyrins and metalloporphyrins," Journal of Porphyrins and Phthalocyanines 05, 225-232 (2001).

${ }^{55}$ C. Neiss, P. Saalfrank, M. Parac, and S. Grimme, "Quantum chemical calculation of excited states of flavin-related molecules," The Journal of Physical Chemistry A 107, 140-147 (2003).

${ }^{56}$ C. Marian, D. Nolting, and R. Weinkauf, "The electronic spectrum of protonated adenine: Theory and experiment," Phys. Chem. Chem. Phys. 7, 3306-3316 (2005).

${ }^{57}$ V. Jovanović, I. Lyskov, M. Kleinschmidt, and C. M. Marian, "On the performance of DFT/MRCI-R and MR-MP2 in spin-orbit coupling calculations on diatomics and polyatomic organic molecules," Molecular Physics 0, 1-29 (2016).

${ }^{58}$ M. Head-Gordon, J. A. Pople, and M. J. Frisch, "MP2 energy evaluation by direct methods," Chemical Physics Letters 153, 503 - 506 (1988).

${ }^{59}$ M. J. Frisch, M. Head-Gordon, and J. A. Pople, "A direct MP2 gradient method," Chemical Physics Letters 166, 275 - 280 (1990).

${ }^{60}$ M. J. Frisch, M. Head-Gordon, and J. A. Pople, "Semi-direct algorithms for the MP2 energy and gradient," Chemical Physics Letters 166, 281 - 289 (1990).

${ }^{61}$ M. Head-Gordon and T. Head-Gordon, "Analytic MP2 frequencies without fifth-order storage. theory and application to bifurcated hydrogen bonds in the water hexamer," Chemical Physics Letters 220, 122 - 128 (1994).

${ }^{62} \mathrm{~F}$. Weigend and R. Ahlrichs, "Balanced basis sets of split valence, triple zeta valence and quadruple zeta valence quality for H to Rn: Design and assessment of accuracy," Phys. 
Chem. Chem. Phys. 7, 3297-3305 (2005).

${ }^{63}$ J. Č́ižek, "On the use of the cluster expansion and the technique of diagrams in calculations of correlation effects in atoms and molecules," in Advances in Chemical Physics (John Wiley \& Sons, Inc., 2007) pp. 35-89.

${ }^{64}$ G. D. Purvis and R. J. Bartlett, "A full coupled-cluster singles and doubles model: The inclusion of disconnected triples," The Journal of Chemical Physics 76, 1910-1918 (1982).

${ }^{65}$ G. E. Scuseria, C. L. Janssen, and H. F. Schaefer, "An efficient reformulation of the closed-shell coupled cluster single and double excitation (CCSD) equations," The Journal of Chemical Physics 89, 7382-7387 (1988).

${ }^{66}$ G. E. Scuseria and H. F. Schaefer, "Is coupled cluster singles and doubles (CCSD) more computationally intensive than quadratic configuration interaction (QCISD)?" The Journal of Chemical Physics 90, 3700-3703 (1989).

${ }^{67}$ A. Dreuw and M. Head-Gordon, "Single-reference ab initio methods for the calculation of excited states of large molecules," Chemical Reviews 105, 4009-4037 (2005).

${ }^{68}$ M. E. Casida, C. Jamorski, K. C. Casida, and D. R. Salahub, "Molecular excitation energies to high-lying bound states from time-dependentdensity-functionalresponsetheory: Characterizationand correctionofthetime-dependentlocaldensityapproximation ionization threshold," The Journal of Chemical Physics 108, 4439-4449 (1998).

${ }^{69}$ A. Dreuw, J. L. Weisman, and M. Head-Gordon, "Long-range charge-transfer excited states in time-dependent density functional theory require non-local exchange," The Journal of Chemical Physics 119, 2943-2946 (2003).

${ }^{70}$ D. J. Tozer, "Relationship between long-range charge-transfer excitation energy error and integer discontinuity in Kohn-Sham theory," The Journal of Chemical Physics 119, 1269712699 (2003).

${ }^{71}$ O. Gritsenko and E. J. Baerends, "Asymptotic correction of the exchange-correlation kernel of time-dependent density functional theory for long-range charge-transfer excitations," The Journal of Chemical Physics 121, 655-660 (2004).

${ }^{72}$ M. J. G. Peach, T. Helgaker, P. Salek, T. W. Keal, O. B. Lutnaes, D. J. Tozer, and N. C. Handy, "Assessment of a Coulomb-attenuated exchange-correlation energy functional," Phys. Chem. Chem. Phys. 8, 558-562 (2006).

${ }^{73}$ M. J. G. Peach, C. R. L. Sueur, K. Ruud, M. Guillaume, and D. J. Tozer, "TDDFT diagnostic testing and functional assessment for triazene chromophores," Phys. Chem. 
Chem. Phys. 11, 4465-4470 (2009).

${ }^{74}$ M. J. G. Peach, P. Benfield, T. Helgaker, and D. J. Tozer, "Excitation energies in density functional theory: An evaluation and a diagnostic test," The Journal of Chemical Physics 128, 044118 (2008).

${ }^{75}$ M. W. Schmidt, K. K. Baldridge, J. A. Boatz, S. T. Elbert, M. S. Gordon, J. H. Jensen, S. Koseki, N. Matsunaga, K. A. Nguyen, S. Su, T. L. Windus, M. Dupuis, and J. A. Montgomery, "General atomic and molecular electronic structure system," Journal of Computational Chemistry 14, 1347-1363 (1993).

${ }^{76}$ M. S. Gordon and M. W. Schmidt, "Chapter 41 - advances in electronic structure theory: GAMESS a decade later," in Theory and Applications of Computational Chemistry, edited by C. E. Dykstra, G. Frenking, K. S. Kim, and G. E. Scuseria (Elsevier, Amsterdam, 2005) pp. $1167-1189$.

${ }^{77}$ S. Grimme, "Density functional calculations with configuration interaction for the excited states of molecules," Chemical Physics Letters 259, 128 - 137 (1996).

${ }^{78}$ A. D. Becke, "A new mixing of Hartree-Fock and local density-functional theories," The Journal of Chemical Physics 98, 1372-1377 (1993).

79 “TURBOMOLE V6.2 2010, a development of University of Karlsruhe and Forschungszentrum Karlsruhe GmbH, 1989-2007, TURBOMOLE GmbH, since 2007; available from http://www. turbomole.com.".

${ }^{80}$ I. Lyskov, M. Kleinschmidt, and C. M. Marian, "Redesign of the DFT/MRCI Hamiltonian," The Journal of Chemical Physics 144, 034104 (2016).

${ }^{81}$ F. Weigend, "Accurate Coulomb-fitting basis sets for H to Rn," Phys. Chem. Chem. Phys. 8, 1057-1065 (2006).

${ }^{82}$ M. J. Frisch, G. W. Trucks, H. B. Schlegel, G. E. Scuseria, M. A. Robb, J. R. Cheeseman, G. Scalmani, V. Barone, B. Mennucci, G. A. Petersson, H. Nakatsuji, M. Caricato, X. Li, H. P. Hratchian, A. F. Izmaylov, J. Bloino, G. Zheng, J. L. Sonnenberg, M. Hada, M. Ehara, K. Toyota, R. Fukuda, J. Hasegawa, M. Ishida, T. Nakajima, Y. Honda, O. Kitao, H. Nakai, T. Vreven, J. A. Montgomery, Jr., J. E. Peralta, F. Ogliaro, M. Bearpark, J. J. Heyd, E. Brothers, K. N. Kudin, V. N. Staroverov, R. Kobayashi, J. Normand, K. Raghavachari, A. Rendell, J. C. Burant, S. S. Iyengar, J. Tomasi, M. Cossi, N. Rega, J. M. Millam, M. Klene, J. E. Knox, J. B. Cross, V. Bakken, C. Adamo, J. Jaramillo, R. Gomperts, R. E. Stratmann, O. Yazyev, A. J. Austin, R. Cammi, C. Pomelli, J. W. 
Ochterski, R. L. Martin, K. Morokuma, V. G. Zakrzewski, G. A. Voth, P. Salvador, J. J. Dannenberg, S. Dapprich, A. D. Daniels, Ö. Farkas, J. B. Foresman, J. V. Ortiz, J. Cioslowski, and D. J. Fox, "Gaussian09 revision E.01," (2009), gaussian Inc. Wallingford CT 2009.

${ }^{83}$ D. J. Tozer and N. C. Handy, "Improving virtual Kohn-Sham orbitals and eigenvalues: Application to excitation energies and static polarizabilities," The Journal of Chemical Physics 109, 10180-10189 (1998).

${ }^{84}$ S. Hirata, C.-G. Zhan, E. Aprà, T. L. Windus, and D. A. Dixon, "A new, self-contained asymptotic correction scheme to exchange-correlation potentials for time-dependent density functional theory," The Journal of Physical Chemistry A 107, 10154-10158 (2003).

${ }^{85}$ E. Fromager, S. Knecht, and H. J. A. Jensen, "Multi-configuration time-dependent density-functional theory based on range separation," The Journal of Chemical Physics 138, 084101 (2013).

${ }^{86}$ M. Hubert, E. D. Hedegard, and H. J. A. Jensen, .

${ }^{87}$ M. Hubert, H. J. A. Jensen, and E. D. Hedegard, . 
UV Excitations of Halons

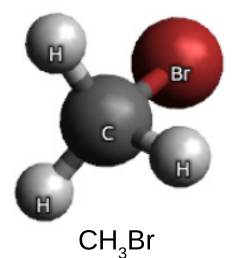

bromomethane (Halon 1001)

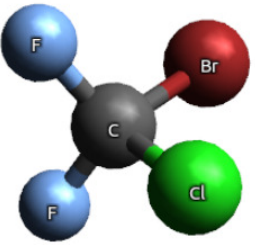

$\mathrm{CBrClF}_{2}$

bromochlorodifluoromethane (Halon 1211)

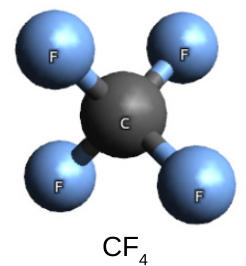

tetrafluoromethane (Halon 14)

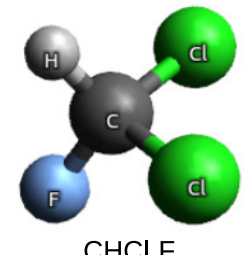

$\mathrm{CHCl}_{2} \mathrm{~F}$

dichlorofluoromethane

(Halon 112)

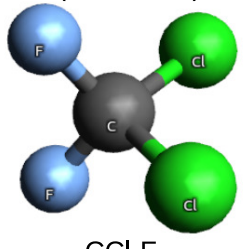

$\mathrm{CCl}_{2} \mathrm{~F}_{2}$

dichlorodifluoromethane

(Halon 122)

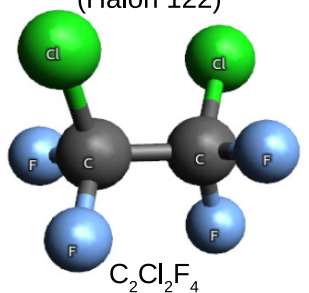

1,2-dichlorotetrafluoroethane (Halon 242)

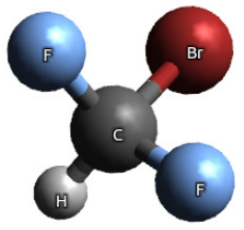

$\mathrm{CHBrF}_{2}$

bromodifluoromethane (Halon 1201)

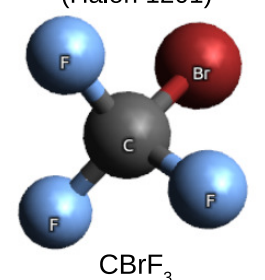

bromotrifluoromethane (Halon 1301)

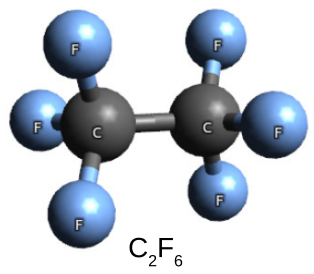

hexafluoroethane (Halon 2600)

FIG. 1. The molecular structures of the nine studied halon molecules (Halons-9 molecular set). 
UV Excitations of Halons
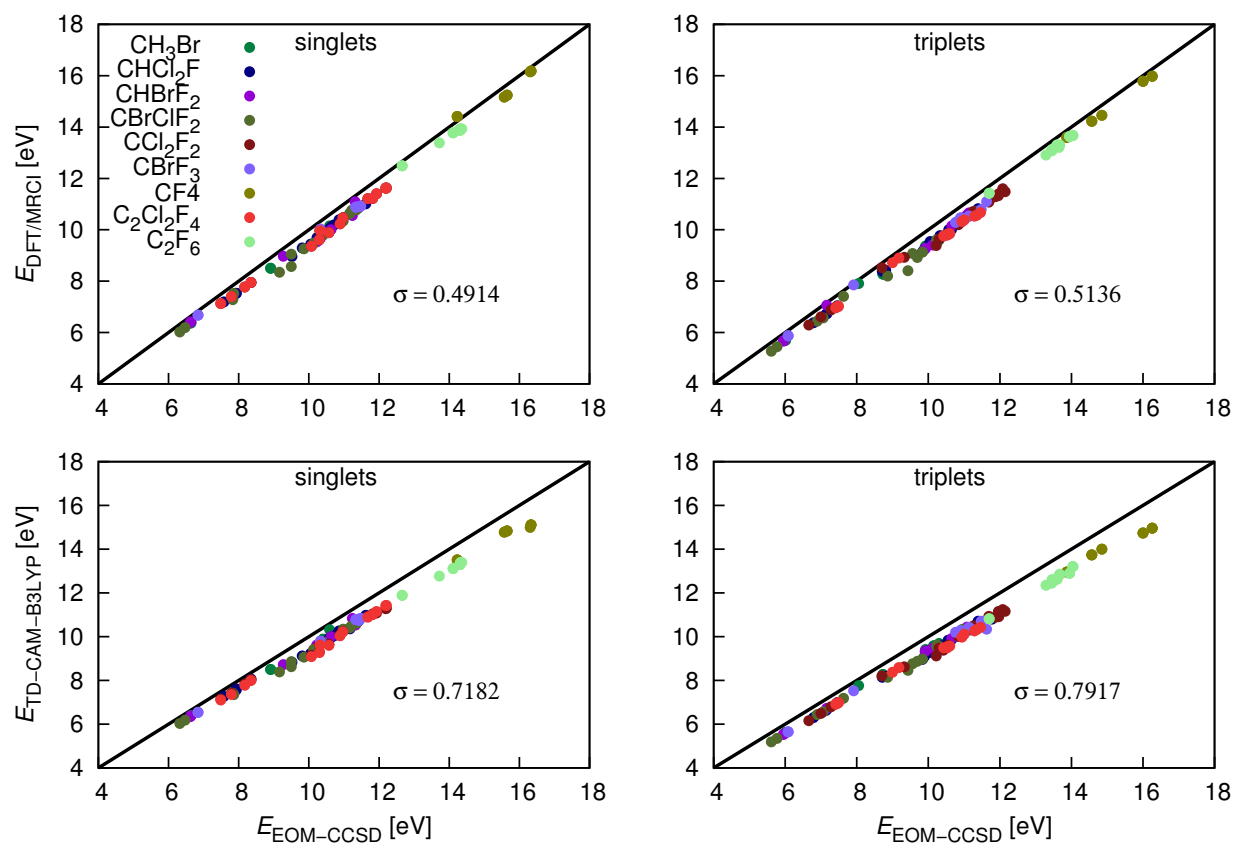

FIG. 2. The correlation diagrams of vertical excitation energies (in eV) for the Halons-9 molecular set obtained with DFT/MRCI and TD-CAM-B3LYP methods, with vertical excitation energies obtained with EOM-CCSD method. 
UV Excitations of Halons

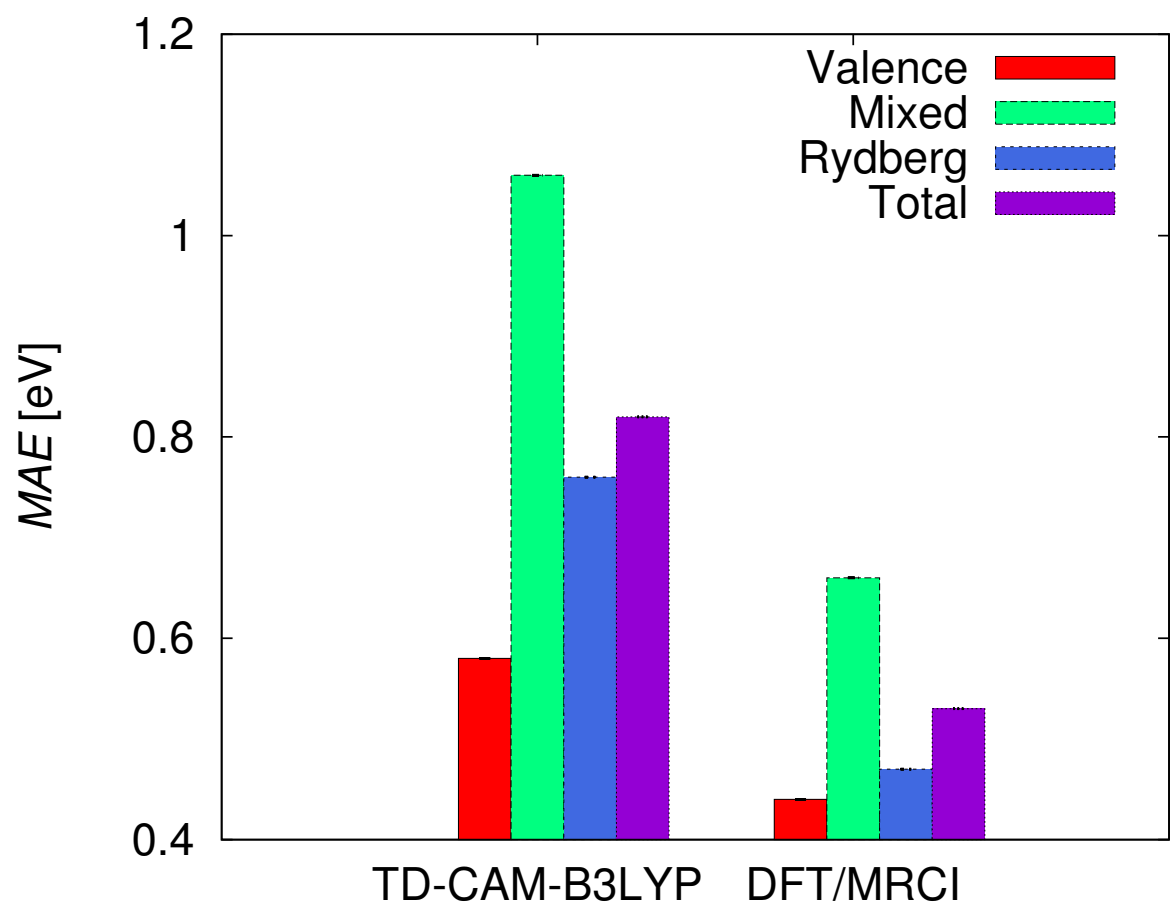

FIG. 3. The mean absolute errors of the vertical excitation energies from the corresponding EOMCCSD values (in eV) of valence, mixed valence-Rydberg, Rydberg, and all studied states of the Halons-9 molecular set obtained with TD-CAM-B3LYP and DFT/MRCI. 
UV Excitations of Halons

TABLE I. Vertical ionization potentials (IP in eV) computed on CCSD/def2-TZVPP level at the MP2/def2-TZVPP optimized geometries and negative values of HOMO energies (- $E_{H O M O}$ in eV) obtained with CAM-B3LYP/def2-TZVPP of the Halons-9 molecular set.

\begin{tabular}{lll}
\hline \hline Molecules & $I P(\mathrm{eV})$ & $-E_{\text {НОмо }}(\mathrm{eV})$ \\
\hline $\mathrm{CH}_{3} \mathrm{Br}$ & 10.54 & 9.29 \\
$\mathrm{CHCl}_{2} \mathrm{~F}$ & 11.86 & 10.54 \\
$\mathrm{CHBrF}_{2}$ & 11.44 & 10.24 \\
$\mathrm{CBrClF}_{2}$ & 11.48 & 10.29 \\
$\mathrm{CCl}_{2} \mathrm{~F}_{2}$ & 12.21 & 10.87 \\
$\mathrm{CBrF}_{3}$ & 11.68 & 8.70 \\
$\mathrm{CF}_{4}$ & 16.60 & 14.24 \\
$\mathrm{C}_{2} \mathrm{Cl}_{2} \mathrm{~F}_{4}$ & 12.60 & 11.24 \\
$\mathrm{C}_{2} \mathrm{~F}_{6}$ & 14.67 & 12.99 \\
\hline
\end{tabular}

TABLE II: Computed vertical excitation energies (eV), oscillator strengths, assignment of the states and $\Lambda$ values for the Halons-9 molecular set.

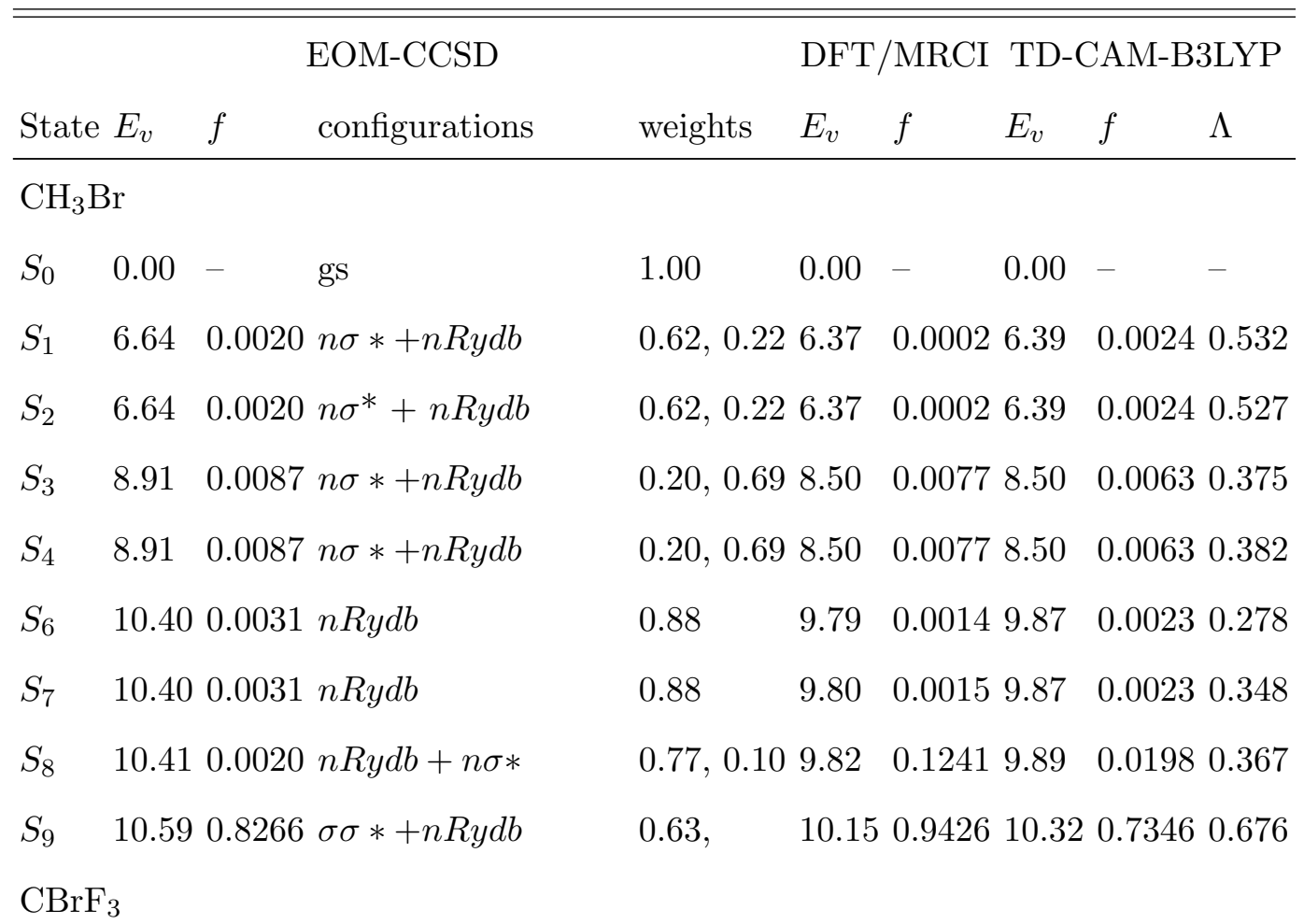


UV Excitations of Halons

\begin{tabular}{|c|c|c|c|c|c|c|c|c|}
\hline$S_{0}$ & 0.00 & gs & 1.00 & 0.00 & - & 0.00 & - & - \\
\hline$S_{1}$ & 6.84 & $0.0000 n \sigma *$ & 0.90 & 6.68 & 0.0014 & 6.54 & 0.0001 & 0.578 \\
\hline$S_{2}$ & 6.84 & $0.0000 n \sigma *$ & 0.90 & 6.68 & 0.0014 & 6.54 & 0.0001 & 0.580 \\
\hline$S_{3}$ & 10.33 & $0.2644 \sigma \sigma *+n R y d b$ & $0.71,0.16$ & 10.03 & 0.2605 & 9.79 & 0.2075 & 0.686 \\
\hline$S_{4}$ & 11.34 & $0.1550 n R y d b$ & 0.84 & 10.88 & 0.1401 & 10.78 & 0.0889 & 0.412 \\
\hline$S_{5}$ & 11.34 & $0.1550 n R y d b$ & 0.84 & 10.88 & 0.1401 & 10.78 & 0.0889 & 0.406 \\
\hline$S_{7}$ & 11.48 & 0.0040 nRydb & 0.77 & 10.92 & 0.0489 & 10.83 & 0.0350 & 0.358 \\
\hline$S_{8}$ & 11.48 & $0.0040 n R y d b$ & 0.77 & 10.92 & 0.0489 & 10.83 & 0.0350 & 0.358 \\
\hline \multicolumn{9}{|c|}{$\mathrm{CF}_{4}$} \\
\hline$S_{0}$ & 0.00 & gs & 1.00 & 0.00 & - & 0.00 & - & - \\
\hline$S_{1}$ & 14.23 & $0.0000 n s$ & 0.75 & 14.41 & 0.0000 & 13.51 & 0.0000 & 0.405 \\
\hline$S_{2}$ & 14.23 & $0.0000 n s$ & 0.75 & 14.41 & 0.0000 & 13.51 & 0.0000 & 0.368 \\
\hline$S_{3}$ & 14.23 & $0.0000 n s$ & 0.75 & 14.41 & 0.0000 & 13.51 & 0.0000 & 0.392 \\
\hline$S_{4}$ & 15.57 & $0.0000 n R y d b+n \sigma *$ & $0.68,0.13$ & 15.17 & 0.0003 & 14.78 & 0.0000 & 0.455 \\
\hline$S_{5}$ & 15.57 & $0.0000 n R y d b+n \sigma *$ & $0.68,0.13$ & 15.17 & 0.0003 & 14.78 & 0.0000 & 0.407 \\
\hline$S_{6}$ & 15.65 & $0.2455 n R y d b$ & 0.68 & 15.24 & 0.2060 & 14.83 & 0.2103 & 0.461 \\
\hline$S_{7}$ & 15.65 & $0.2455 n R y d b$ & 0.68 & 15.24 & 0.2060 & 14.83 & 0.2103 & 0.476 \\
\hline$S_{8}$ & 15.65 & $0.2455 n R y d b$ & 0.68 & 15.24 & 0.2060 & 14.83 & 0.2103 & 0.473 \\
\hline \multicolumn{9}{|c|}{$\mathrm{CHCl}_{2} \mathrm{~F}$} \\
\hline$S_{0}$ & 0.00 & gs & 1.00 & 0.00 & - & 0.00 & - & - \\
\hline$S_{1}$ & 7.58 & $0.0159 n \sigma *$ & 0.82 & 7.19 & 0.0149 & 7.27 & 0.0147 & 0.596 \\
\hline$S_{3}$ & 7.93 & $0.0038 n \sigma *+n R y d b$ & & 7.53 & 0.0028 & 7.61 & 0.0005 & 0.563 \\
\hline$S_{4}$ & 8.35 & $0.0007 n \sigma *+n R y d b$ & & 7.95 & 0.0013 & 8.06 & 0.0005 & 0.574 \\
\hline$S_{5}$ & 9.52 & $0.0017 n R y d b+n \sigma *$ & & 8.96 & 0.0011 & 8.83 & 0.0002 & 0.565 \\
\hline$S_{6}$ & 9.81 & $0.0569 n R y d b+n \sigma *$ & & 9.29 & 0.0758 & 9.12 & 0.0389 & 0.584 \\
\hline$S_{7}$ & 10.05 & $0.0156 n \sigma *$ & & 9.44 & 0.0084 & 9.24 & 0.0085 & 0.562 \\
\hline$S_{8}$ & 10.23 & $0.0056 n \sigma *$ & & 9.69 & 0.0044 & 9.60 & 0.0025 & 0.376 \\
\hline$S_{9}$ & 10.54 & $0.0104 n \sigma *$ & & 9.96 & 0.0542 & 9.78 & 0.0128 & 0.515 \\
\hline$S_{10}$ & 10.58 & $0.0129 n \sigma *$ & & 10.04 & 0.0243 & 9.94 & 0.0095 & 0.469 \\
\hline$S_{11}$ & 10.64 & $0.0348 n \sigma *$ & & 10.12 & 0.0028 & 9.97 & 0.0095 & 0.489 \\
\hline
\end{tabular}


UV Excitations of Halons

$S_{12} \quad 10.720 .0011 n \sigma *$

$S_{13} \quad 10.860 .0026 n \sigma *$

$S_{15} \quad 11.170 .0125 n \sigma *+\sigma \sigma *$

$S_{17} \quad 11.620 .7122 n \sigma *+\sigma \sigma *$

$\mathrm{CHBrF}_{2}$

$S_{0} \quad 0.00 \quad-\quad$ gs

$S_{1} \quad 6.620 .0012 n \sigma^{*}$

$S_{3} \quad 9.27 \quad 0.2670 \sigma \sigma^{*}$

$S_{4} \quad 10.220 .0317 n \sigma^{*}$

$S_{5} \quad 10.270 .0146 n \sigma^{*}$

$S_{6} \quad 10.330 .0114 n \sigma^{*}$

$S_{7} \quad 10.630 .1441 n \sigma^{*}$

$S_{8} \quad 11.240 .0107 n \sigma *+\sigma \sigma *+n R y d b$

$S_{9} \quad 11.320 .0170 n \sigma *+\sigma \sigma *+n R y d b$

$S_{10} 11.330 .0424 n \sigma *+\sigma \sigma^{*}$
$S_{14} \quad 10.980 .1553 n \sigma *$

$S_{16} \quad 11.410 .0381 n \sigma *+\sigma \sigma *$

$10.190 .006410 .04 \quad 0.0006 \quad 0.381$

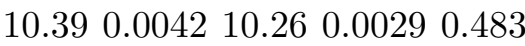

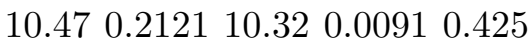

$10.64 \quad 0.0157 \quad 10.36 \quad 0.10590 .554$

10.850 .043410 .700 .02710 .412

$\begin{array}{lllll}11.01 & 0.9102 & 10.98 & 0.4998 & 0.489\end{array}$

1.00

0.81

0.71

0.80

0.81

0.82

0.64

$0.00-$

$0.00-$

$\begin{array}{lllll}6.39 & 0.0022 & 6.34 & 0.0014 & 0.565\end{array}$

$\begin{array}{lllll}8.97 & 0.2732 & 8.72 & 0.2130 & 0.662\end{array}$

$\begin{array}{lllll}9.53 & 0.0340 & 9.60 & 0.0273 & 0.318\end{array}$

$\begin{array}{lllll}9.58 & 0.0148 & 9.62 & 0.0157 & 0.238\end{array}$

$\begin{array}{llllll}9.67 & 0.0013 & 9.70 & 0.0037 & 0.327\end{array}$

10.000 .185310 .000 .09050 .465

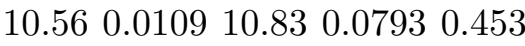

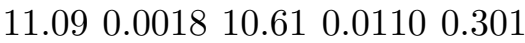

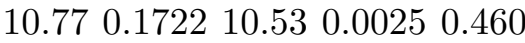

$\mathrm{CBrClF}_{2}$

$\begin{array}{lll}S_{0} & 0.00 \quad-\quad \mathrm{g}\end{array}$

$S_{1} \quad 6.32 \quad 0.0095 n \sigma^{*}$

1.00

$0.00-$

$0.00-$

0.86

$\begin{array}{lllll}6.02 & 0.0088 & 6.02 & 0.0089 & 0.602\end{array}$

$\begin{array}{lll}S_{4} & 7.86 & 0.0247 n \sigma^{*}\end{array}$

0.81

0.77

$\begin{array}{llllll}7.52 & 0.0206 & 7.48 & 0.0196 & 0.580\end{array}$

$S_{5} \quad 9.16 \quad 0.0846 n \sigma^{*}$

0.84

$\begin{array}{lll}S_{6} & 9.50 & 0.0012 n \sigma^{*}\end{array}$

$\begin{array}{lllll}8.35 & 0.0172 & 8.35 & 0.0157 & 0.524\end{array}$

$S_{7} \quad 9.50 \quad 0.1985 n \sigma *+\sigma \sigma^{*}$

$S_{9} \quad 10.130 .0554 n \sigma^{*}$

0.72

$S_{11} 10.980 .1863 n \sigma *+n R y d b+\sigma \sigma^{*}$

$S_{12} \quad 11.160 .1566 n \sigma *+n R y d b+\sigma \sigma^{*}$

$S_{13} \quad 11.220 .0915 n \sigma *+n R y d b+\sigma \sigma^{*}$

$S_{14} \quad 11.360 .0905$ ns

0.82

$\mathrm{CCl}_{2} \mathrm{~F}_{2}$ $\begin{array}{lllll}8.57 & 0.0000 & 8.59 & 0.0005 & 0.404\end{array}$

$\begin{array}{llllll}9.05 & 0.3428 & 8.84 & 0.1751 & 0.639\end{array}$

$\begin{array}{lllll}9.47 & 0.0840 & 9.37 & 0.0778 & 0.580\end{array}$

10.340 .350410 .310 .10840 .586

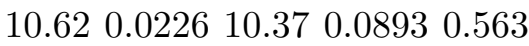

$10.760 .078210 .490 .0008 \quad 0.246$

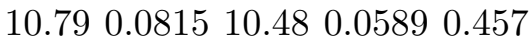


UV Excitations of Halons

\begin{tabular}{|c|c|c|c|c|c|c|c|}
\hline$S_{0}$ & 0.00 & gs & 1.00 & 0.00 & - & 0.00 & - \\
\hline$S_{1}$ & 7.49 & $0.0171 n \sigma^{*}$ & 0.88 & 7.13 & 0.0146 & 7.10 & 0.01500 .611 \\
\hline$S_{6}$ & 10.30 & $0.0011 n \sigma^{*}$ & 0.84 & 9.62 & 0.0000 & 9.27 & 0.00050 .568 \\
\hline$S_{8}$ & 10.31 & $0.2091(n+\sigma) \sigma *+n R y d b$ & $0.79,0.08$ & 9.97 & 0.2481 & 9.62 & 0.11280 .624 \\
\hline$S_{9}$ & 10.89 & $0.0442 n \sigma *+n R y d b$ & $0.68,0.16$ & 10.23 & 0.1152 & 10.04 & 0.06750 .583 \\
\hline$S_{11}$ & 11.68 & $0.0150(n+\sigma) \sigma+n R y d b$ & $0.41,0.40$ & 11.21 & 0.0089 & 10.89 & 0.01590 .682 \\
\hline$S_{12}$ & 11.82 & $0.1841 n R y d b+n \sigma^{*}$ & $0.69,0.10$ & 11.22 & 0.3770 & 11.00 & 0.19270 .537 \\
\hline$S_{13}$ & 11.93 & $0.1942 n R y d b$ & 0.81 & 11.40 & 0.0110 & 11.05 & 0.00260 .260 \\
\hline$S_{15}$ & 12.21 & $0.0040 n R y d b$ & 0.74 & 11.63 & 0.0000 & 11.27 & 0.00520 .616 \\
\hline \multicolumn{8}{|c|}{$\mathrm{C}_{2} \mathrm{~F}_{6}$} \\
\hline$S_{0}$ & 0.00 & gs & 1.00 & 0.00 & - & 0.00 & - \\
\hline$S_{1}$ & 12.66 & $0.0427(n+\sigma) \sigma^{*}$ & 0.84 & 12.49 & 0.0663 & 11.89 & 0.03540 .631 \\
\hline$S_{2}$ & 12.66 & $0.0427(n+\sigma) \sigma^{*}$ & 0.84 & 12.49 & 0.0661 & 11.89 & 0.03540 .643 \\
\hline$S_{4}$ & 14.11 & $0.0278 n \sigma^{*}$ & 0.76 & 13.77 & 0.0182 & 13.11 & 0.02490 .495 \\
\hline$S_{5}$ & 14.11 & $0.0278 n \sigma^{*}$ & 0.76 & 13.78 & 0.0220 & 13.11 & 0.02490 .477 \\
\hline$S_{8}$ & 14.31 & $0.2143 n \sigma *+n s$ & $0.58,0.14$ & 13.92 & 0.0000 & 13.36 & 0.00000 .410 \\
\hline \multicolumn{8}{|c|}{$\mathrm{C}_{2} \mathrm{Cl}_{2} \mathrm{~F}_{4}$} \\
\hline$S_{0}$ & 0.00 & gs & 1.00 & 0.00 & - & 0.00 & - \\
\hline$S_{1}$ & 8.29 & $0.0009 n \sigma^{*}$ & & 7.81 & 0.0003 & 7.88 & 0.00060 .582 \\
\hline$S_{3}$ & 8.38 & $0.0020 n \sigma^{*}$ & & 7.91 & 0.0033 & 8.01 & 0.00010 .600 \\
\hline$S_{5}$ & 10.34 & $0.0863 n \sigma^{*}+\sigma \sigma^{*}$ & & 9.9 & 0.0715 & 9.39 & 0.04560 .609 \\
\hline$S_{6}$ & 10.38 & $0.1039 n \sigma^{*}+\sigma \sigma^{*}$ & & 9.84 & 0.0909 & 9.45 & 0.05020 .604 \\
\hline$S_{7}$ & 10.75 & $0.0034 n R y d b$ & & 10.05 & 0.0024 & 9.65 & 0.00250 .588 \\
\hline$S_{8}$ & 10.87 & $0.0052 n R y d b$ & & 10.14 & 0.0044 & 9.68 & 0.00040 .596 \\
\hline$S_{9}$ & 11.09 & $0.0462 n R y d b$ & & 10.46 & 0.0676 & 9.98 & 0.03640 .606 \\
\hline$S_{10}$ & 11.17 & $0.0447 n R y d b$ & & 10.52 & 0.0577 & 10.18 & 0.03440 .622 \\
\hline$S_{11}$ & 11.37 & $0.0020 n R y d b$ & & 10.77 & 0.0040 & 10.41 & $0.0045 \quad 0.427$ \\
\hline$S_{13}$ & 11.62 & $0.0016 n R y d b+n \sigma^{*}$ & & 10.92 & 0.0144 & 10.73 & 0.00300 .498 \\
\hline$S_{14}$ & 11.66 & $0.0183 n R y d b$ & & 10.97 & 0.0115 & 10.82 & 0.00070 .459 \\
\hline$S_{15}$ & 11.89 & $0.0003 n R y d b$ & & 11.12 & 0.0004 & 10.89 & 0.00370 .552 \\
\hline
\end{tabular}


UV Excitations of Halons

\begin{tabular}{|c|c|c|}
\hline$S_{16}$ & 11.950 .0076 nRydb & 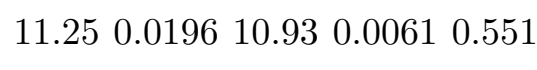 \\
\hline$S_{17}$ & $12.060 .0052 n R y d b+n \sigma^{*}$ & 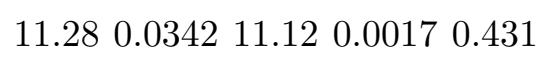 \\
\hline$S_{19}$ & $12.190 .0053 n R y d b$ & $\begin{array}{lllll}11.72 & 0.0225 & 11.38 & 0.0046 & 0.537\end{array}$ \\
\hline$S_{20}$ & $12.500 .1489 n R y d b$ & $\begin{array}{lllll}12.02 & 0.1056 & 11.44 & 0.0001 & 0.342\end{array}$ \\
\hline
\end{tabular}


UV Excitations of Halons

TABLE III: Computed vertical excitation energies (eV), assignment of the states and $\Lambda$ values of the triplet excited states for the Halons-9 molecular set.

\begin{tabular}{|c|c|c|c|c|c|c|}
\hline & & EOM-CCSD & & DFT/MRCI & I TD-C & CAM-B3LYP \\
\hline Stat & $E_{v}$ & configurations & s weights & $E_{v}$ & $E_{v}$ & $\Lambda$ \\
\hline $\mathrm{CH}_{3}$ & & & & & & \\
\hline$T_{1}$ & 6.01 & $n \sigma^{*}$ & 0.87 & 5.70 & 5.62 & 0.529 \\
\hline$T_{2}$ & 6.01 & $n \sigma^{*}$ & 0.87 & 5.70 & 5.62 & 0.529 \\
\hline$T_{3}$ & 8.05 & $\sigma \sigma^{*}$ & 0.80 & 7.91 & 7.76 & 0.702 \\
\hline$T_{4}$ & 8.73 & $n \sigma^{*}$ & 0.89 & 8.27 & 8.27 & 0.377 \\
\hline$T_{5}$ & 8.73 & $n \sigma^{*}$ & 0.89 & 8.27 & 8.27 & 0.384 \\
\hline $\mathrm{CBr}$ & & & & & & \\
\hline$T_{1}$ & 6.08 & $n \sigma^{*}$ & 0.90 & 5.87 & 5.65 & 0.575 \\
\hline$T_{2}$ & 6.08 & $n \sigma^{*}$ & 0.90 & 5.87 & 5.65 & 0.578 \\
\hline$T_{3}$ & 7.91 & $\sigma \sigma^{*}$ & 0.87 & 7.85 & 7.52 & 0.725 \\
\hline$T_{4}$ & 10.76 & $n p$ & 0.76 & 10.29 & 10.20 & 0.539 \\
\hline$T_{5}$ & 10.90 & $n s$ & 0.80 & 10.46 & 10.28 & 0.407 \\
\hline $\mathrm{CF}_{4}$ & & & & & & \\
\hline$T_{1}$ & 13.87 & $n s+n \sigma^{*}$ & $0.60,0.11$ & 13.60 & 12.95 & 0.415 \\
\hline$T_{2}$ & 13.87 & $n s+n \sigma^{*}$ & $0.60,0.11$ & 13.60 & 12.95 & 50.384 \\
\hline$T_{3}$ & 13.87 & $n s+n \sigma^{*}$ & $0.60,0.11$ & 13.60 & 12.95 & 0.391 \\
\hline$T_{4}$ & 14.57 & $n R y d b+n \sigma^{*}$ & $0.54,0.31$ & 14.23 & 13.74 & 40.445 \\
\hline$T_{5}$ & 14.57 & $n R y d b+n \sigma^{*}$ & $0.54,0.31$ & 14.23 & 13.74 & 0.470 \\
\hline $\mathrm{CHC}$ & $\mathrm{l}_{2} \mathrm{~F}$ & & & & & \\
\hline$T_{1}$ & 6.79 & $n \sigma^{*}$ & & 6.38 & 6.30 & 0.591 \\
\hline$T_{2}$ & 7.14 & $n \sigma^{*}$ & & 6.71 & 6.63 & 0.612 \\
\hline$T_{3}$ & 7.17 & $n \sigma^{*}$ & & 6.74 & 6.68 & 0.564 \\
\hline$T_{4}$ & 7.46 & $n \sigma^{*}$ & & 7.04 & 6.99 & 0.571 \\
\hline$T_{5}$ & 8.71 & $n \sigma^{*}+\sigma \sigma^{*}$ & & 8.39 & 8.14 & 0.571 \\
\hline $\mathrm{CHB}$ & & & & & & \\
\hline
\end{tabular}


UV Excitations of Halons

$\begin{array}{lllllll}T_{1} & 5.95 & n \sigma^{*} & 0.82 & 5.66 & 5.53 & 0.564 \\ T_{2} & 5.96 & n \sigma^{*} & 0.81 & 5.69 & 5.55 & 0.543 \\ T_{3} & 7.16 & \sigma \sigma^{*} & 0.86 & 7.06 & 6.72 & 0.693 \\ T_{4} & 9.91 & n \sigma^{*} & 0.76 & 9.25 & 9.33 & 0.348 \\ T_{5} & 9.92 & n \sigma^{*} & 0.78 & 9.28 & 9.34 & 0.450\end{array}$

$\mathrm{CBrClF}_{2}$

$\begin{array}{lllllll}T_{1} & 5.61 & n \sigma^{*} & 0.97 & 5.27 & 5.19 & 0.601 \\ T_{2} & 5.77 & n \sigma^{*} & 0.86 & 5.45 & 5.35 & 0.549 \\ T_{3} & 6.89 & n \sigma^{*} & 0.83 & 6.44 & 6.44 & 0.591 \\ T_{4} & 7.06 & n \sigma^{*} & 0.89 & 6.57 & 6.56 & 0.465 \\ T_{5} & 7.63 & n \sigma^{*}+\sigma \sigma^{*} & & 7.41 & 7.18 & 0.618\end{array}$

$\mathrm{CCl}_{2} \mathrm{~F}_{2}$

$\begin{array}{lllllll}T_{1} & 6.66 & n \sigma^{*} & 0.90 & 6.29 & 6.16 & 0.610 \\ T_{2} & 7.00 & n \sigma^{*} & 0.90 & 6.61 & 6.49 & 0.567 \\ T_{3} & 7.28 & n \sigma^{*} & 0.90 & 6.88 & 6.79 & 0.567 \\ T_{4} & 7.42 & n \sigma^{*} & 0.87 & 7.00 & 6.93 & 0.597 \\ T_{5} & 8.70 & n \sigma^{*}+\sigma \sigma^{*} & & 8.52 & 8.21 & 0.597 \\ \mathrm{C}_{2} \mathrm{~F}_{6} & & & & & \\ T_{1} & 11.70(n+\sigma) \sigma^{*} & 0.89 & 11.42 & 10.82 & 0.633 \\ T_{2} & 11.70(n+\sigma) \sigma^{*} & 0.89 & 11.42 & 10.82 & 0.645 \\ T_{3} & 13.29 & n s & 0.65 & 12.91 & 12.35 & 0.471 \\ T_{4} & 13.46 & n \sigma *+n s & 0.49,0.11 & 13.08 & 12.61 & 0.491 \\ T_{5} & 13.45 n \sigma^{*} & 0.67 & 13.11 & 12.45 & 0.483\end{array}$

$\mathrm{C}_{2} \mathrm{Cl}_{2} \mathrm{~F}_{4}$

\begin{tabular}{llllll}
$T_{1}$ & 7.41 & $n \sigma^{*}$ & 6.96 & 6.91 & 0.587 \\
$T_{2}$ & 7.45 & $n \sigma^{*}$ & 7.00 & 6.95 & 0.598 \\
$T_{3}$ & 7.48 & $n \sigma^{*}$ & 7.01 & 6.97 & 0.600 \\
$T_{4}$ & 7.49 & $n \sigma^{*}$ & 7.03 & 6.99 & 0.586 \\
$T_{5}$ & 9.00 & $n \sigma^{*}+\sigma \sigma^{*}$ & 8.72 & 8.37 & 0.652 \\
\hline
\end{tabular}


UV Excitations of Halons

TABLE IV. Mean error (ME), mean absolute error (MAE), standard deviation (SD) and maximum and minimum deviations (in eV) of the DFT/MRCI and TD-CAM-B3LYP excitation energies from EOM-CCSD results for the Halons-9 molecular set.

\begin{tabular}{|c|c|c|c|}
\hline & & TD-CAM-B3LYP & DFT-MRCI \\
\hline \multirow[t]{5}{*}{ Valence (64 states) } & $\mathrm{ME}$ & -0.58 & -0.44 \\
\hline & MAE & 0.58 & 0.44 \\
\hline & $\mathrm{SD}$ & 0.63 & 0.48 \\
\hline & Max. error & 1.10 & 1.02 \\
\hline & Min. error & 0.27 & 0.06 \\
\hline \multirow[t]{5}{*}{ Mixed (85 states) } & $\mathrm{ME}$ & -1.06 & -0.66 \\
\hline & MAE & 1.06 & 0.66 \\
\hline & $\mathrm{SD}$ & 0.80 & 0.51 \\
\hline & Max. error & 1.33 & 0.80 \\
\hline & Min. error & 0.28 & 0.18 \\
\hline \multirow[t]{5}{*}{ Rydberg (91 states) } & $\mathrm{ME}$ & -0.76 & -0.47 \\
\hline & MAE & 0.76 & 0.47 \\
\hline & $\mathrm{SD}$ & 0.85 & 0.54 \\
\hline & Max. error & 1.20 & 0.80 \\
\hline & Min. error & 0.51 & 0.19 \\
\hline \multirow[t]{5}{*}{ Total (240 states) } & $\mathrm{ME}$ & -0.82 & -0.53 \\
\hline & MAE & 0.82 & 0.53 \\
\hline & $\mathrm{SD}$ & 0.78 & 0.51 \\
\hline & Max. error & 1.33 & 1.02 \\
\hline & Min. error & 0.27 & 0.06 \\
\hline
\end{tabular}


UV Excitations of Halons

TABLE V. Computed vertical excitation energies $\left(\mathrm{E}_{v}\right.$ in $\left.\mathrm{eV}\right)$ compared to the corresponding positions of the band maxima in the available experimental UV photoabsorption spectra of the Halons-9 set. The computed oscillator strengths for the transitions are also given (f).

\begin{tabular}{|c|c|c|c|c|c|c|c|c|}
\hline \multirow{2}{*}{ Molecule } & \multirow{2}{*}{ State } & \multicolumn{2}{|c|}{ EOM-CCSD } & \multicolumn{2}{|c|}{$\mathrm{DFT} / \mathrm{MRCI}$} & \multicolumn{2}{|c|}{ TD-CAM-B3LYP } & \multirow{2}{*}{$\begin{array}{l}\text { Exp. } \\
\mathrm{E}_{v}\end{array}$} \\
\hline & & $\mathrm{E}_{v}$ & $\mathrm{f}$ & $\mathrm{E}_{v}$ & $\mathrm{f}$ & $\mathrm{E}_{v}$ & $\mathrm{f}$ & \\
\hline \multirow{2}{*}{$\mathrm{CH}_{3} \mathrm{Br}$} & $T_{1}$ & 6.01 & - & 5.70 & - & 5.62 & - & $5.98^{19}$ \\
\hline & $S_{1}$ & 6.64 & 0.0020 & 6.37 & 0.0002 & 6.39 & 0.0024 & $6.37^{19}$ \\
\hline \multirow{2}{*}{$\mathrm{CBrF}_{3}$} & $T_{1}$ & 6.08 & - & 5.878 & - & 5.65 & - & \multirow{2}{*}{$6.05^{20}$} \\
\hline & $S_{1}$ & 6.84 & 0.0000 & 6.68 & 0.0014 & 6.54 & 0.0001 & \\
\hline \multirow{2}{*}{$\mathrm{CF}_{4}$} & $T_{1}$ & 13.87 & - & 13.60 & - & 12.95 & - & \multirow{2}{*}{$13.6,13.9^{22}$} \\
\hline & $S_{1}$ & 14.23 & 0.0000 & 14.41 & 0.0000 & 13.51 & 0.0000 & \\
\hline \multirow{2}{*}{$\mathrm{CHCl}_{2} \mathrm{~F}$} & $T_{1}$ & 6.79 & - & 6.38 & - & 6.30 & - & \multirow{2}{*}{$7.29^{21}$} \\
\hline & $S_{1}$ & 7.58 & 0.0159 & 7.19 & 0.0149 & 7.27 & 0.0147 & \\
\hline \multirow{2}{*}{$\mathrm{CHBrF}_{2}$} & $T_{1}$ & 5.95 & - & 5.66 & - & 5.53 & - & \multirow{2}{*}{$6.52^{21}$} \\
\hline & $S_{1}$ & 6.62 & 0.0012 & 6.39 & 0.0022 & 6.34 & 0.0014 & \\
\hline \multirow{2}{*}{$\mathrm{CBrClF}_{2}$} & $T_{1}$ & 5.61 & - & 5.27 & - & 5.19 & - & \multirow[t]{2}{*}{$6.02^{21}$} \\
\hline & $S_{1}$ & 6.32 & 0.0095 & 6.02 & 0.0088 & 6.03 & 0.0089 & \\
\hline \multirow{2}{*}{$\mathrm{CCl}_{2} \mathrm{~F}_{2}$} & $T_{1}$ & 6.66 & - & 6.29 & - & 6.19 & - & \multirow{2}{*}{$6.97^{24}$} \\
\hline & $S_{1}$ & 7.49 & 0.0171 & 7.13 & 0.0146 & 7.10 & 0.0150 & \\
\hline \multirow{2}{*}{$\mathrm{C}_{2} \mathrm{~F}_{6}$} & $T_{1}$ & 10.70 & - & 11.42 & - & 10.82 & - & \multirow{2}{*}{$12.1^{28}$} \\
\hline & $S_{1}$ & 12.66 & 0.0427 & 12.49 & 0.0663 & 11.89 & 0.0354 & \\
\hline \multirow{2}{*}{$\mathrm{C}_{2} \mathrm{Cl}_{2} \mathrm{~F}_{4}$} & $T_{1}$ & 7.41 & - & 6.96 & - & 6.91 & - & \multirow{2}{*}{$7.21^{29}$} \\
\hline & $S_{1}$ & 8.29 & 0.0009 & 7.81 & 0.0003 & 7.88 & 0.0006 & \\
\hline
\end{tabular}

